Supporting Information

\title{
A Facile One-Pot Preparation of Potassium Hydroxyaryl- and
}

\section{(Hydroxyalkyl)aryltrifluoroborates}

Young Hee Park, ${ }^{\dagger}$, Hong Ryul Ahn, ${ }^{\dagger}$ Belgin Canturk,,${ }^{\S}$ Sang Il Jeon, ${ }^{\dagger}$ Seokjoon Lee, Heonjoong Kang, ${ }^{*, \perp}$ Gary A. Molander, ${ }^{*}$, and Jungyeob Ham ${ }^{*}, \dagger$

${ }^{\dagger}$ Korea Institute of Science and Technology, 290 Daejeon-dong, Gangneung 210-340, Korea

${ }^{\S}$ Roy and Diana Vagelos Laboratories, Department of Chemistry University of Pennsylvania, Philadelphia, Pennsylvania 19104-6323,USA

${ }^{\perp}$ Center for Marine Natural Products and Drug Discovery, School of Earth and Environmental Sciences, Seoul National University, NS-80, Seoul 151-747, Korea

${ }^{\ddagger}$ Department of Chemistry, Kangnung National University, Gangneung 210-702, Korea

"Department of Basic Science, Kwandong University College of Medicine, Gangneung 210-701, Korea

General Considerations......................................................... 2 Preparation of Potassium Hydroxyaryl- and (Hydroxyalkyl)aryltrifluoroborates.. .................................................................................. Microwave-promoted Suzuki-Miyaura Cross-Coupling Reaction ..................s8 Suzuki-Miyaura Cross-Coupling Reaction.......................................S8

References..................................................................S11

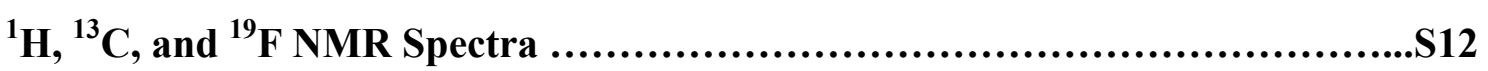


General Considerations: ${ }^{1} \mathrm{H},{ }^{13} \mathrm{C}$, and ${ }^{19} \mathrm{~F}$ NMR spectra were recorded at 500, 126, and $376 \mathrm{MHz}$, respectively. ${ }^{19} \mathrm{~F}$ NMR chemical shifts were referenced to external $\mathrm{CFCl}_{3}(0.0$ ppm). Mass spectra of potassium organotrifluoroborates were performed using negative ion electrospray mass spectrometry. Microwave reactions ${ }^{1}$ were carried out in a monomode microwave apparatus (CEM Discover $\left.{ }^{\circledR}\right)$. Reactions were performed in glass vessels (capacity $10 \mathrm{~mL}$ ) sealed with a septum. Temperature measurements were conducted using an infrared temperature sensor positioned below the reaction vessel.

\section{$\begin{array}{lllll}\text { Preparation of } & \text { Potassium } & \text { Hydroxyaryl- and }\end{array}$ (Hydroxyalkyl)aryltrifluoroborates}

\section{General Procedure}

To a soln of bromophenol (1.0 mmol) in THF $(5 \mathrm{~mL})$ was slowly added $t$-BuLi (1.7 M soln in hexane, $1.77 \mathrm{~mL}, 3.0 \mathrm{mmol}$ ) at $-78{ }^{\circ} \mathrm{C}$ under $\mathrm{N}_{2}$. The soln was vigorously stirred for $1 \mathrm{~h}$ (ortho-halogen substituted starting materials were stirred for $3 \mathrm{~h}$ ). To this mixture was slowly added triisopropyl borate $(188 \mathrm{mg}, 1.0 \mathrm{mmol})$ at $-78{ }^{\circ} \mathrm{C}$. After 30 min at $-30{ }^{\circ} \mathrm{C}$, the reaction mixture was quenched with $1 \mathrm{~N} \mathrm{KHF}_{2}(5.0 \mathrm{~mL}, 5.0 \mathrm{mmol})$ and then warmed to rt. After stirring for $30 \mathrm{~min}$, the suspension was concentrated on a rotary evaporator. The residual white solid was dissolved in dry acetone $(7.0 \mathrm{~mL})$, and the insoluble salts were filtered off. The resulting organic soln was concentrated on a rotary evaporator. The crude product was purified by dissolution in acetone and precipitation with $\mathrm{Et}_{2} \mathrm{O}$ to obtain the desired compound.<smiles>Oc1ccc(Br)cc1</smiles>

\section{Potassium 4-hydroxyphenyltrifluoroborate (1)}

The desired compound was prepared using 4-bromophenol (173 mg, $1.0 \mathrm{mmol})$ by the general procedure (a white solid, $122 \mathrm{mg}, 61 \%$ yield). $\mathrm{mp}=248{ }^{\circ} \mathrm{C}$ (dec.). ${ }^{1} \mathrm{H}$ NMR $\left(500 \mathrm{MHz}\right.$, Acetone- $d_{6}+$ DMSO- $\left.d_{6}\right) \delta 7.98($ br s, $1 \mathrm{H}), 7.29$ (d, 2H, $\left.J=7.5 \mathrm{~Hz}\right), 6.60$ (d, $2 \mathrm{H}, J=8.0 \mathrm{~Hz}) .{ }^{13} \mathrm{C}$ NMR $\left(126 \mathrm{MHz}\right.$, Acetone- $\left.d_{6}+\mathrm{DMSO}-d_{6}\right) \delta 155.6,132.7,113.5$. ${ }^{19} \mathrm{~F}\left(376 \mathrm{MHz}, \mathrm{CD}_{3} \mathrm{OD}\right) \delta$-141.1. FT-IR (KBr): 3643, 3530, 3436, 3168, 1633, 1209, 960, $831 \mathrm{~cm}^{-1}$. HRMS: $m / z$ calcd for $\mathrm{C}_{6} \mathrm{H}_{5} \mathrm{BF}_{3} \mathrm{O}\left[\mathrm{M}-\mathrm{K}^{+}\right]$161.0386, found 161.0388 . 
<smiles>Oc1ccc(Br)cc1</smiles>

\section{Potassium 4-hydroxyphenyltrifluoroborate (1)}

The desired compound was prepared using 4-iodophenol $(220 \mathrm{mg}, 1.0 \mathrm{mmol})$ by the general procedure (196 mg, 98\% yield).<smiles>Oc1cccc(Br)c1</smiles>

\section{Potassium 3-hydroxyphenyltrifluoroborate (2)}

The desired compound was prepared using 3-bromophenol (173 $\mathrm{mg}, 1.0 \mathrm{mmol}$ ) by the general procedure (56 mg, 28\% yield). mp $>250{ }^{\circ} \mathrm{C} .{ }^{1} \mathrm{H} \mathrm{NMR}\left(500 \mathrm{MHz}, \mathrm{CD}_{3} \mathrm{OD}\right) \delta$ $7.04(\mathrm{t}, 1 \mathrm{H}, J=7.5 \mathrm{~Hz}), 6.98(\mathrm{~m}, 2 \mathrm{H}), 6.61(\mathrm{dd}, 1 \mathrm{H}, J=7.5,2.5 \mathrm{~Hz}) .{ }^{13} \mathrm{C}$ NMR $(126$ $\left.\mathrm{MHz}, \mathrm{CD}_{3} \mathrm{OD}\right) \delta 155.8,127.9,122.8,117.9,113.0 .{ }^{19} \mathrm{~F}\left(376 \mathrm{MHz}, \mathrm{CD}_{3} \mathrm{OD}\right) \delta-$ 141.9. FT-IR (KBr): 3447, 1628, 1264, 1077, 850, $746 \mathrm{~cm}^{-1}$. HRMS: $\mathrm{m} / z$ calcd for $\mathrm{C}_{6} \mathrm{H}_{5} \mathrm{BF}_{3} \mathrm{O}\left[\mathrm{M}-\mathrm{K}^{+}\right]$161.0386, found 161.0387.<smiles>Oc1cccc(Br)c1</smiles>

\section{Potassium 3-hydroxyphenyltrifluoroborate (2)}

The desired compound was prepared using 3-iodophenol (220 mg, $1.0 \mathrm{mmol})$ by the general procedure (196 mg, 98\% yield).<smiles>Oc1ccccc1Br</smiles>

\section{Potassium 2-hydroxyphenyltrifluoroborate (3) ${ }^{2}$}

The desired compound was prepared using 2-bromophenol (173 mg, $1.0 \mathrm{mmol})$ by the general procedure (42 mg, 21\% yield). $\mathrm{mp}>250{ }^{\circ} \mathrm{C} .{ }^{1} \mathrm{H}$ NMR $\left(500 \mathrm{MHz}, \mathrm{DMSO}-d_{6}\right) \delta$ 7.37 (q, 1H, -OH, $J=9.1 \mathrm{~Hz}), 7.11(\mathrm{dd}, 1 \mathrm{H}, J=7.0,1.0 \mathrm{~Hz}), 6.91(\mathrm{td}, 1 \mathrm{H}, J=7.6,1.6$ $\mathrm{Hz}), 6.58(\mathrm{t}, 1 \mathrm{H}, J=7.1 \mathrm{~Hz}), 6.49(\mathrm{~d}, 1 \mathrm{H}, J=8.0 \mathrm{~Hz}) .{ }^{13} \mathrm{C}$ NMR $\left(126 \mathrm{MHz}\right.$, DMSO- $\left.d_{6}\right)$ $\delta 159.9,133.6,127.6,119.0,114.0 .{ }^{19} \mathrm{~F}\left(376 \mathrm{MHz}, \mathrm{DMSO}-d_{6}\right) \delta-133.7$. FT-IR (KBr): 3418, 1524, 1141, 969, $757 \mathrm{~cm}^{-1}$. HRMS: $\mathrm{m} / z$ calcd for $\mathrm{C}_{6} \mathrm{H}_{5} \mathrm{BF}_{3} \mathrm{O}\left[\mathrm{M}-\mathrm{K}^{+}\right]$161.0386, found 161.0391 . 
<smiles>Oc1ccccc1Br</smiles>

\section{Potassium 2-hydroxyphenyltrifluoroborate (3)}

The desired compound was prepared using 2-iodophenol $(220 \mathrm{mg}, 1.0 \mathrm{mmol})$ by the general procedure (154 mg, 77\% yield).<smiles>Cc1cc(Br)ccc1O</smiles>

\section{Potassium 4-hydroxy-3-methylphenyltrifluoroborate (4)}

The desired compound was prepared using 4-bromo-2-methylphenol (187 mg, 1.0 $\mathrm{mmol})$ by the general procedure $(163 \mathrm{mg}, 76 \%$ yield $) . \mathrm{mp}=188{ }^{\circ} \mathrm{C} .{ }^{1} \mathrm{H}$ NMR $(500 \mathrm{MHz}$, Acetone- $\left.d_{6}\right) \delta 7.36(\mathrm{~s}, 1 \mathrm{H}), 7.19(\mathrm{~s}, 1 \mathrm{H}), 7.12(\mathrm{~d}, 1 \mathrm{H}, J=7.5 \mathrm{~Hz}), 6.57(\mathrm{~d}, 1 \mathrm{H}, J=7.5$ $\mathrm{Hz}), 2.15(\mathrm{~s}, 3 \mathrm{H}) .{ }^{13} \mathrm{C}$ NMR $\left(126 \mathrm{MHz}\right.$, Acetone- $\left.d_{6}\right) \delta 153.4,134.6,130.2,121.3,113.2$, 15.7. ${ }^{19} \mathrm{~F}\left(376 \mathrm{MHz}\right.$, Acetone- $\left.d_{6}\right) \delta$-140.2. FT-IR (KBr): 3480, 2921, 1654, 1603, 1488 , 1301, 1154, 1053, 981, 928, $877 \mathrm{~cm}^{-1}$. HRMS: $\mathrm{m} / z$ calcd for $\mathrm{C}_{7} \mathrm{H}_{7} \mathrm{BF}_{3} \mathrm{O}\left[\mathrm{M}-\mathrm{K}^{+}\right]$ 175.0542, found 175.0542 .<smiles>Cc1cc(Br)ccc1O</smiles>

\section{Potassium 4-hydroxy-3-methylphenyltrifluoroborate (4)}

The desired compound was prepared using 4-iodo-2-methylphenol (234 mg, $1.0 \mathrm{mmol}$ ) by the general procedure ( $208 \mathrm{mg}, 97 \%$ yield).<smiles>Cc1cc(Br)cc(C)c1O</smiles>

\section{Potassium 4-hydroxy-3,5-dimethylphenyltrifluoroborate (5)}

The desired compound was prepared using 4-bromo-2,6-dimethylphenol (201 mg, 1.0 $\mathrm{mmol})$ by the general procedure $(148 \mathrm{mg}, 65 \%$ yield $) . \mathrm{mp}=196{ }^{\circ} \mathrm{C} .{ }^{1} \mathrm{H}$ NMR $(500 \mathrm{MHz}$, Acetone- $\left.d_{6}\right) \delta 6.89(\mathrm{~s}, 2 \mathrm{H}), 6.33(\mathrm{~s}, 1 \mathrm{H}), 2.02(\mathrm{~s}, 6 \mathrm{H}) .{ }^{13} \mathrm{C}$ NMR (126 MHz, Acetone- $\left.d_{6}\right)$ $\delta 151.2,132.3,121.1,114.9,16.0 .{ }^{19} \mathrm{~F}\left(376 \mathrm{MHz}\right.$, Acetone- $\left.d_{6}\right) \delta-140.3$. FT-IR (KBr): 3616, 3336, 3030, 1610, 1464, 1161, 1275, 1124, 957, $812 \mathrm{~cm}^{-1}$. HRMS: $m / z$ calcd for $\mathrm{C}_{8} \mathrm{H}_{9} \mathrm{BF}_{3} \mathrm{O}\left[\mathrm{M}-\mathrm{K}^{+}\right] 189.0699$, found 189.0698 . 
<smiles>Oc1ccc(Br)cc1Cl</smiles>

\section{Potassium 3-chloro-4-hydroxyphenyltrifluoroborate (6)}

The desired compound was prepared using 4-bromo-2-chlorophenol (208 mg, 1.0 mmol) by the general procedure $(209 \mathrm{mg}, 89 \%$ yield $) . \mathrm{mp}=263{ }^{\circ} \mathrm{C} .{ }^{1} \mathrm{H}$ NMR $(500 \mathrm{MHz}$, Acetone- $\left.d_{6}\right) \delta 7.89$ (br s, 1H), $7.36(\mathrm{~s}, 1 \mathrm{H}), 7.22(\mathrm{~d}, 1 \mathrm{H}, J=7.5 \mathrm{~Hz}), 6.77(\mathrm{~d}, 1 \mathrm{H}, J=7.5$ $\mathrm{Hz}) .{ }^{13} \mathrm{C}$ NMR $\left(126 \mathrm{MHz}\right.$, Acetone- $\left.d_{6}\right) \delta 150.5,132.9,131.3,118.7,115.3 .{ }^{19} \mathrm{~F}(376$ $\mathrm{MHz}$, Acetone- $\left.d_{6}\right) \delta$-141.1. FT-IR (KBr): 3469, 3045, 1608, 1508, 1391, 1216, 991, 819, $711 \mathrm{~cm}^{-1}$. HRMS: $\mathrm{m} / z$ calcd for $\mathrm{C}_{6} \mathrm{H}_{4} \mathrm{ClBF}_{3} \mathrm{O}\left[\mathrm{M}-\mathrm{K}^{+}\right]$194.9996, found 194.9989 .<smiles>Oc1ccc(-c2ccc(Br)cc2)cc1</smiles>

\section{Potassium 4'-hydroxy-[1,1'-biphenyl]-4-trifluoroborate (7)}

The desired compound was prepared using 4'-bromo-[1,1'-biphenyl]-4-ol (249 mg, 1.0 mmol) by the general procedure (102 mg, 37\% yield). mp $>250{ }^{\circ} \mathrm{C} .{ }^{1} \mathrm{H}$ NMR $(500 \mathrm{MHz}$, Acetone- $d_{6}+$ DMSO- $\left.d_{6}\right) \delta 8.99(\mathrm{br} \mathrm{s}, 1 \mathrm{H}), 7.52(\mathrm{~d}, 2 \mathrm{H}, J=8.0 \mathrm{~Hz}), 7.42(\mathrm{~d}, 2 \mathrm{H}, J=8.5$ $\mathrm{Hz}), 7.32(\mathrm{~d}, 2 \mathrm{H}, J=7.5 \mathrm{~Hz}), 6.86(\mathrm{~d}, 2 \mathrm{H}, J=8.5 \mathrm{~Hz}) .{ }^{13} \mathrm{C}$ NMR $\left(126 \mathrm{MHz}\right.$, Acetone- $d_{6}$ + DMSO- $\left.d_{6}\right) \delta 157.0,137.7,133.6,132.3,127.6,124.4,115.7 .{ }^{19} \mathrm{~F}\left(376 \mathrm{MHz}, \mathrm{CD}_{3} \mathrm{OD}\right)$ $\delta$-140.4. FT-IR (KBr): $3446,1628,1082 \mathrm{~cm}^{-1}$. HRMS: $\mathrm{m} / z$ calcd for $\mathrm{C}_{12} \mathrm{H}_{9} \mathrm{BF}_{3} \mathrm{O}[\mathrm{M}-$ $\left.\mathrm{K}^{+}\right]$237.0699, found, 237.0697 .<smiles>Oc1ccc(-c2ccc(Br)cc2)cc1</smiles>

\section{Potassium 4'-hydroxy-[1,1'-biphenyl]-4-trifluoroborate (7)}

The desired compound was prepared using 4'-iodo-[1,1'-biphenyl]-4-ol (296 mg, 1.0 $\mathrm{mmol}$ ) by the general procedure ( $238 \mathrm{mg}, 86 \%$ yield).<smiles>Oc1ccc2cc(Br)ccc2c1</smiles> 


\section{Potassium 6-hydroxy-2-naphthalenetrifluoroborate (8)}

The desired compound was prepared using 6-bromo-2-naphthol (223 mg, $1.0 \mathrm{mmol}$ ) by the general procedure (190 mg, 76\% yield). $\mathrm{mp}>250{ }^{\circ} \mathrm{C}$. ${ }^{1} \mathrm{H}$ NMR $(500 \mathrm{MHz}$, Acetone$\left.d_{6}\right) \delta 8.19(\mathrm{~s}, 1 \mathrm{H}), 7.84(\mathrm{~s}, 1 \mathrm{H}), 7.60(\mathrm{~d}, 1 \mathrm{H}, J=3.7 \mathrm{~Hz}), 7.59(\mathrm{~d}, 1 \mathrm{H}, J=8.1 \mathrm{~Hz}), 7.43$ $(\mathrm{d}, 1 \mathrm{H}, J=8.1 \mathrm{~Hz}), 7.07(\mathrm{~d}, 1 \mathrm{H}, J=2.4 \mathrm{~Hz}), 6.99(\mathrm{dd}, 1 \mathrm{H}, J=8.8,2.5 \mathrm{~Hz}) .{ }^{13} \mathrm{C} \mathrm{NMR}$ $\left(126 \mathrm{MHz}\right.$, Acetone- $\left.d_{6}\right) \delta 154.0,134.1,131.6,130.1,129.3,128.8,123.7,116.8,108.8$. ${ }^{19} \mathrm{~F}\left(376 \mathrm{MHz}\right.$, Acetone- $\left.d_{6}\right) \delta-140.9$. FT-IR (KBr): 3622, 3331, 1681, 1630, 1482, 1251, 1190, 1004, 907, $862 \mathrm{~cm}^{-1}$. HRMS: $\mathrm{m} / z$ calcd for $\mathrm{C}_{10} \mathrm{H}_{7} \mathrm{BF}_{3} \mathrm{O}\left[\mathrm{M}-\mathrm{K}^{+}\right]$ 211.0542, found 211.0540 .<smiles>OCc1ccccc1Br</smiles>

\section{Potassium 2-(hydroxymethyl)phenyltrifluoroborate (9)}

The desired compound was prepared using 2-bromo-benzyl alcohol (187 mg, $1.0 \mathrm{mmol})$ by the general procedure $(128 \mathrm{mg}, 60 \%$ yield $) . \mathrm{mp}=286{ }^{\circ} \mathrm{C}(\mathrm{dec}.) .{ }^{1} \mathrm{H}$ NMR $(500 \mathrm{MHz}$, Acetone- $\left.d_{6}\right) \delta 7.54(\mathrm{~m}, 1 \mathrm{H}), 7.11(\mathrm{~m}, 1 \mathrm{H}), 7.01(\mathrm{~m}, 2 \mathrm{H}), 4.64(\mathrm{~s}, 2 \mathrm{H}), 3.63(\mathrm{br} \mathrm{s}, 1 \mathrm{H})$. ${ }^{13} \mathrm{C}$ NMR $\left(500 \mathrm{MHz}\right.$, Acetone- $\left.d_{6}\right) \delta 145.2,132.6,127.3,125.4,125.3,65.8 .{ }^{19} \mathrm{~F}(376$ $\mathrm{MHz}$, Acetone- $\left.d_{6}\right) \delta$-137.3. FT-IR (KBr): 3446, 1637, 1362, 1215, 1184, 1044, 986, $747 \mathrm{~cm}^{-1}$. HRMS: $m / z$ calcd for $\mathrm{C}_{7} \mathrm{H}_{7} \mathrm{BF}_{3} \mathrm{O}\left[\mathrm{M}-\mathrm{K}^{+}\right]$175.0542, found 175.0542 .<smiles>OCc1cccc(Br)c1</smiles>

\section{Potassium 3-(hydroxymethyl)phenyltrifluoroborate (10)}

The desired compound was prepared using 3-bromo-benzyl alcohol (187 mg, $1.0 \mathrm{mmol})$ by the general procedure $(178 \mathrm{mg}, 83 \%$ yield $) . \mathrm{mp}=161{ }^{\circ} \mathrm{C} .{ }^{1} \mathrm{H}$ NMR $(500 \mathrm{MHz}$, Acetone- $\left.d_{6}\right) \delta 7.45(\mathrm{~s}, 1 \mathrm{H}), 7.36(\mathrm{t}, 1 \mathrm{H}, J=4.5 \mathrm{~Hz}), 7.07(\mathrm{~d}, 2 \mathrm{H}, J=4.5 \mathrm{~Hz}), 4.53(\mathrm{~d}$, $2 \mathrm{H}, J=6.0 \mathrm{~Hz}), 3.82(\mathrm{t}, 1 \mathrm{H}, J=6.0 \mathrm{~Hz}) \cdot{ }^{13} \mathrm{C} \mathrm{NMR}\left(126 \mathrm{MHz}\right.$, Acetone- $\left.d_{6}\right) \delta 139.8$, $130.6,130.5,126.3,124.1,65.2 .{ }^{19} \mathrm{~F}\left(376 \mathrm{MHz}\right.$, Acetone- $\left.d_{6}\right) \delta$-141.1. FT-IR (KBr): $3535,2916,1637,1418,1388,1248,1158,981,957,781 \mathrm{~cm}^{-1}$. HRMS: $m / z$ calcd for $\mathrm{C}_{7} \mathrm{H}_{7} \mathrm{BF}_{3} \mathrm{O}\left[\mathrm{M}-\mathrm{K}^{+}\right]$175.0542, found 175.0540 .<smiles>OCc1ccc(Br)cc1</smiles>

Potassium 4-(hydroxymethyl)phenyltrifluoroborate (11) 
The desired compound was prepared using 4-bromo-benzyl alcohol (187 mg, $1.0 \mathrm{mmol})$ by the general procedure $(193 \mathrm{mg}, 90 \%$ yield $) . \mathrm{mp}=211{ }^{\circ} \mathrm{C} .{ }^{1} \mathrm{H}$ NMR $(500 \mathrm{MHz}$, Acetone- $\left.d_{6}\right) \delta 7.44(\mathrm{~d}, 2 \mathrm{H}, J=7.5), 7.10(\mathrm{~d}, 2 \mathrm{H}, J=7.5 \mathrm{~Hz}), 4.54(\mathrm{~d}, 2 \mathrm{H}, J=6.0 \mathrm{~Hz})$, $3.83(\mathrm{t}, 1 \mathrm{H}, J=6.0 \mathrm{~Hz}) .{ }^{13} \mathrm{C}$ NMR $\left(126 \mathrm{MHz}\right.$, Acetone- $\left.d_{6}\right) \delta 139.0,131.6,125.2,64.8$. ${ }^{19} \mathrm{~F}\left(376 \mathrm{MHz}\right.$, Acetone- $\left.d_{6}\right) \delta$-142.2. FT-IR (KBr): 3588, 3512, 3425, 2934, 1647, 1399 , 1226, 967, 842, $803 \mathrm{~cm}^{-1}$. HRMS: $m / z$ calcd for $\mathrm{C}_{7} \mathrm{H}_{7} \mathrm{BF}_{3} \mathrm{O}\left[\mathrm{M}-\mathrm{K}^{+}\right] 175.0542$, found 175.0545 .<smiles>OCCc1ccccc1Br</smiles>

\section{Potassium 2-(2-hydroxyethyl)phenyltrifluoroborate (12)}

The desired compound was prepared using 2-bromo-phenethyl alcohol (201 mg, 1.0 mmol) by the general procedure (116 mg, 51\% yield). $\mathrm{mp}=257{ }^{\circ} \mathrm{C} .{ }^{1} \mathrm{H} \mathrm{NMR}(500 \mathrm{MHz}$, Acetone- $\left.d_{6}\right) \delta 7.43(\mathrm{~m}, 1 \mathrm{H}), 6.97(\mathrm{~m}, 2 \mathrm{H}), 6.88(\mathrm{~m}, 1 \mathrm{H}), 3.90(\mathrm{t}, 2 \mathrm{H}, J=5.5 \mathrm{~Hz}), 2.65(\mathrm{t}$, $2 \mathrm{H}, J=5.5 \mathrm{~Hz}) .{ }^{13} \mathrm{C}$ NMR $\left(126 \mathrm{MHz}\right.$, Acetone- $\left.d_{6}\right) \delta 143.2,131.4,126.0,125.4,124.7$, 61.7, 34.1. ${ }^{19} \mathrm{~F}$ (376 MHz, Acetone- $\left.d_{6}\right) \delta$-140.8. FT-IR (KBr): 3421, 3059, 2928, 2882, $1598,1443,1276,1221,1094,995,893,750 \mathrm{~cm}^{-1}$. HRMS: $m / z$ calcd for $\mathrm{C}_{8} \mathrm{H}_{9} \mathrm{BF}_{3} \mathrm{O}$ $\left[\mathrm{M}-\mathrm{K}^{+}\right]$189.0699, found 189.0703 .<smiles>OCCc1cccc(Br)c1</smiles>

\section{Potassium 3-(2-hydroxyethyl)phenyltrifluoroborate (13)}

The desired compound was prepared using 3-bromo-phenethyl alcohol (201 mg, 1.0 $\mathrm{mmol})$ by the general procedure $(155 \mathrm{mg}, 68 \%$ yield $) . \mathrm{mp}=136{ }^{\circ} \mathrm{C} .{ }^{1} \mathrm{H}$ NMR $(500 \mathrm{MHz}$, Acetone- $\left.d_{6}\right) \delta 7.37(\mathrm{~s}, 1 \mathrm{H}), 7.33(\mathrm{~d}, 1 \mathrm{H}, J=7.5 \mathrm{~Hz}), 7.04(\mathrm{t}, 1 \mathrm{H}, J=7.5 \mathrm{~Hz}), 6.94(\mathrm{~d}$, $1 \mathrm{H}, J=7.5 \mathrm{~Hz}), 3.71(\mathrm{q}, 2 \mathrm{H}, J=7.0 \mathrm{~Hz}), 3.58(\mathrm{t}, 1 \mathrm{H}, J=5.5 \mathrm{~Hz}), 2.75(\mathrm{t}, 2 \mathrm{H}, J=7.0$ $\mathrm{Hz}) .{ }^{13} \mathrm{C}$ NMR $\left(126 \mathrm{MHz}\right.$, Acetone- $\left.d_{6}\right) \delta 136.8,132.5,129.6,126.6,126.2,63.8,40.2$. ${ }^{19} \mathrm{~F}\left(376 \mathrm{MHz}\right.$, Acetone- $\left.d_{6}\right) \delta$-141.1. FT-IR (KBr): 3493, 3034, 2901, 1633, 1414, 1303 , 1242, 1160, 1026, 950, 903, 791, $717 \mathrm{~cm}^{-1}$. HRMS: $m / z$ calcd for $\mathrm{C}_{8} \mathrm{H}_{9} \mathrm{BF}_{3} \mathrm{O}\left[\mathrm{M}-\mathrm{K}^{+}\right]$ 189.0699, found 189.0705 .<smiles>OCCc1ccc(Br)cc1</smiles>

Potassium 4-(2-hydroxyethyl)phenyltrifluoroborate (14) 
The desired compound was prepared using 4-bromo-phenethyl alcohol (201 mg, 1.0 mmol) by the general procedure (166 mg, 73\% yield). $\mathrm{mp}=194{ }^{\circ} \mathrm{C} .{ }^{1} \mathrm{H}$ NMR $(500 \mathrm{MHz}$, Acetone- $\left.d_{6}\right) \delta 7.40(\mathrm{~d}, 2 \mathrm{H}, J=7.5 \mathrm{~Hz}), 6.98(\mathrm{~d}, 2 \mathrm{H}, J=7.5 \mathrm{~Hz}), 3.70(\mathrm{q}, 2 \mathrm{H}, J=7.5$ $\mathrm{Hz}), 3.54(\mathrm{t}, 1 \mathrm{H}, J=5.5 \mathrm{~Hz}), 2.74(\mathrm{t}, 2 \mathrm{H}, J=7.5 \mathrm{~Hz}) .{ }^{13} \mathrm{C}$ NMR (126 MHz, Acetone- $\left.d_{6}\right)$ $\delta 135.9,131.8,127.2,63.8,39.9 .{ }^{19} \mathrm{~F}$ (376 MHz, Acetone- $\left.d_{6}\right) \delta$-141.2. FT-IR (KBr): 3437, 2952, 1613, 1398, 1229, 1011, 963, 834, $812 \mathrm{~cm}^{-1}$ HRMS: $\mathrm{m} / z$ calcd for $\mathrm{C}_{8} \mathrm{H}_{9} \mathrm{BF}_{3} \mathrm{O}\left[\mathrm{M}-\mathrm{K}^{+}\right]$189.0699, found 189.0699.

\section{Microwave-promoted Suzuki-Miyaura Cross-Coupling Reaction ${ }^{3}$}

General Procedure. To a $16 \times 90 \mathrm{~mm}$ glass vessel containing a stirring bar were added the potassium hydroxyaryl- or (hydroxyalkyl)aryltrifluoroborate $(0.1 \mathrm{mmol}), \mathrm{K}_{2} \mathrm{CO}_{3}$ (41.4 mg, $0.3 \mathrm{mmol}), \mathrm{Pd}(\mathrm{OAc})_{2}\left(0.11 \mathrm{mg}, 5 \times 10^{-4} \mathrm{mmol}, 0.5 \mathrm{~mol} \%\right),{ }^{3}$ and aryl bromides $(0.1 \mathrm{mmol})$. The mixture was dissolved in methanol $(1.0 \mathrm{~mL})$. The vessel was sealed with a septum and placed into the microwave cavity. An initial microwave irradiation of $100 \mathrm{~W}$ was used, with the temperature being ramped from $\mathrm{rt}$ to the desired temperature of $80{ }^{\circ} \mathrm{C}$. Once this temperature was reached, the reaction mixture was held at this temperature with stirring for $5 \mathrm{~min}$ (typically, the irradiation power was $100 \mathrm{~W}$ during the warm up period and $3-5 \mathrm{~W}$, on average, during the constant phase). The reaction mixture was cooled to room temperature. The solvent was removed under vacuum. The residual compound was dissolved in ethyl acetate $(2.0 \mathrm{~mL})$, and the insoluble salts were filtered off through a small amount of silica gel. The resulting organic soln was concentrated on a rotary evaporator. The crude product was purified by preparative TLC $(0.5 \mathrm{~mm})$.

\section{Suzuki-Miyaura Cross-Coupling Reaction ${ }^{4}$}

General Procedure. To a $10 \times 75 \mathrm{~mm}$ test tube were added the potassium (hydroxyalkyl)aryltrifluoroborate $(0.1 \mathrm{mmol}), \mathrm{K}_{2} \mathrm{CO}_{3}(41.4 \mathrm{mg}, 0.3 \mathrm{mmol}), \mathrm{Pd}(\mathrm{OAc})_{2}$ $\left(0.11 \mathrm{mg}, 5 \times 10^{-4} \mathrm{mmol}, 0.5 \mathrm{~mol} \%\right)$, and aryl bromides $(0.1 \mathrm{mmol})$ with a stirring bar. Methanol $(1.0 \mathrm{~mL})$ was added and the mixture was heated in an oil bath at $80{ }^{\circ} \mathrm{C}$. The reaction was monitored by TLC. After $1 \mathrm{~h}$, the aryl bromide was totally consumed, and the reaction mixture was cooled to room temperature. The solvent was removed under vacuum. The residual compound was dissolved in ethyl acetate $(2.0 \mathrm{~mL})$, and the insoluble salts were filtered off through a small amount of silica gel. The resulting organic soln was concentrated on a rotary evaporator. The crude product was purified by preparative TLC $(0.5 \mathrm{~mm})$. 
<smiles>Cc1ccc(-c2ccc3cc(O)ccc3c2)cc1</smiles>

\section{6-p-Tolyl-naphthalen-2-ol (15)}

The desired compound was prepared with potassium 6-hydroxy-2naphthalenetrifluoroborate $(25 \mathrm{mg}, 0.1 \mathrm{mmol})$ and 4-bromotoluene (17.1 mg, $0.1 \mathrm{mmol})$ using the general procedure $(19.4 \mathrm{mg}$, a white solid, $83 \%$ yield $) . \mathrm{mp}=139{ }^{\circ} \mathrm{C} .{ }^{1} \mathrm{H}$ NMR (500 MHz, $\left.\mathrm{CDCl}_{3}\right) \delta 7.91($ br s, $1 \mathrm{H}), 7.75(\mathrm{~d}, 1 \mathrm{H}, J=9.0 \mathrm{~Hz}), 7.70(\mathrm{~d}, 1 \mathrm{H}, J=8.0$ $\mathrm{Hz}), 7.65(\mathrm{dd}, 1 \mathrm{H}, J=8.0,2.5 \mathrm{~Hz}), 7.55(\mathrm{~d}, 2 \mathrm{H}, J=8.0 \mathrm{~Hz}), 7.24(\mathrm{~d}, 2 \mathrm{H}, J=7.8 \mathrm{~Hz})$, $7.12(\mathrm{~d}, 1 \mathrm{H}, J=2.5 \mathrm{~Hz}), 7.08(\mathrm{dd}, 1 \mathrm{H}, J=9.0,2.5 \mathrm{~Hz}), 4.89(\mathrm{~s}, 1 \mathrm{H}), 2.40(\mathrm{~s}, 3 \mathrm{H}) .{ }^{13} \mathrm{C}$ NMR $\left(126 \mathrm{MHz}, \mathrm{CDCl}_{3}\right) \delta 153.5,138.4,137.1,136.6,133.8,130.3,129.8,127.2,127.0$, 126.4, 125.5, 124.0, 118.3, 109.5, 21.3. FT-IR (KBr): 3400, 2915, 1630, 1603, 1506, 1441, 1237, 1199, 1152, 865, $803 \mathrm{~cm}^{-1}$. ESI-MS: $m / z$ calcd for $\mathrm{C}_{17} \mathrm{H}_{13} \mathrm{O}\left[\mathrm{M}-\mathrm{H}^{+}\right] 233.09$, found 233.09.<smiles>Cc1ccc(-c2ccc(CO)cc2)cc1</smiles>

\section{(4'-Methylbiphenyl-4-yl)-methanol (16)}

The desired compound was prepared with potassium 4(hydroxymethyl)phenyltrifluoroborate $(21.4 \mathrm{mg}, 0.1 \mathrm{mmol})$ and 4-bromotoluene (17.1 $\mathrm{mg}, 0.1 \mathrm{mmol})$ using the general procedure (18.2 $\mathrm{mg}$, a white solid, $92 \%$ yield). $\mathrm{mp}=$ $119{ }^{\circ} \mathrm{C} .{ }^{1} \mathrm{H}$ NMR $\left(500 \mathrm{MHz}, \mathrm{CDCl}_{3}\right) \delta 7.54(\mathrm{~d}, 2 \mathrm{H}, J=8.0 \mathrm{~Hz}), 7.45(\mathrm{~d}, 2 \mathrm{H}, J=8.0$ $\mathrm{Hz}), 7.38(\mathrm{~d}, 2 \mathrm{H}, J=8.0 \mathrm{~Hz}), 7.21(\mathrm{~d}, 2 \mathrm{H}, J=8.0 \mathrm{~Hz}), 4.70$ (d, 2H, $J=5.0 \mathrm{~Hz}), 2.38(\mathrm{~s}$, $3 \mathrm{H}), 1.70(\mathrm{t}, 1 \mathrm{H}, J=5.5 \mathrm{~Hz}) .{ }^{13} \mathrm{C}$ NMR $\left(126 \mathrm{MHz} \mathrm{CDCl}_{3}\right) \delta 140.8,139.8,138.1,137.3$, 129.7, 127.7, 127.3, 127.1, 65.4, 21.3. FT-IR (KBr): 3332, 2914, 2855, 1638, 1499, 1396, 1049, 1013, $799 \mathrm{~cm}^{-1}$. ESI-MS: $\mathrm{m} / z$ calcd for $\mathrm{C}_{14} \mathrm{H}_{13} \mathrm{O}\left[\mathrm{M}-\mathrm{H}^{+}\right] 197.09$, found 197.10.<smiles>Cc1ccc(-c2ccc(CCO)cc2)cc1</smiles> 
The desired compound was prepared with potassium 4-(2hydroxyethyl)phenyltrifluoroborate $(22.8 \mathrm{mg}, 0.1 \mathrm{mmol})$ and 4-bromotoluene $(17.1 \mathrm{mg}$, $0.1 \mathrm{mmol})$ using the general procedure ( $18.7 \mathrm{mg}$, a white solid, $88 \%$ yield). $\mathrm{mp}=95{ }^{\circ} \mathrm{C}$. ${ }^{1} \mathrm{H} \mathrm{NMR}\left(500 \mathrm{MHz}, \mathrm{CDCl}_{3}\right) \delta 7.49(\mathrm{~d}, 2 \mathrm{H}, J=8.0 \mathrm{~Hz}), 7.44(\mathrm{~d}, 2 \mathrm{H}, J=8.0 \mathrm{~Hz}), 7.25$ (d, $2 \mathrm{H}, J=8.0 \mathrm{~Hz}), 7.20(\mathrm{~d}, 2 \mathrm{H}, J=8.0 \mathrm{~Hz}), 3.88(\mathrm{q}, 2 \mathrm{H}, J=6.5 \mathrm{~Hz}), 2.89$ (t, $2 \mathrm{H}, J=7.0$ $\mathrm{Hz}), 2.37$ (s, 3H), 1.43 (t, 1H, $J=5.5 \mathrm{~Hz}) .{ }^{13} \mathrm{C} \mathrm{NMR}\left(126 \mathrm{MHz}, \mathrm{CDCl}_{3}\right) \delta 139.6,138.2$, 137.4, 137.1, 129.7, 129.6, 127.4, 127.1, 63.9, 39.0, 21.3. FT-IR (KBr): 3415, 2921, 1616, 1500, 1046, $806 \mathrm{~cm}^{-1}$. ESI-MS: $m / z$ calcd for $\mathrm{C}_{15} \mathrm{H}_{15} \mathrm{O}\left[\mathrm{M}-\mathrm{H}^{+}\right] 211.11$, found 211.11.

(cN

\section{(4'-Cyanobiphenyl-4-yl)-methanol (18)}

The desired compound was prepared with potassium 4-(2hydroxymethyl)phenyltrifluoroborate $(21.4 \mathrm{mg}, 0.1 \mathrm{mmol})$ and 4-bromobenzonitrile (18.2 $\mathrm{mg}, 0.1 \mathrm{mmol})$ using the general procedure (19.6 mg, a white solid, 94\% yield). $\mathrm{mp}=151{ }^{\circ} \mathrm{C} .{ }^{1} \mathrm{H}$ NMR $\left(500 \mathrm{MHz}, \mathrm{CDCl}_{3}\right) \delta 7.69(\mathrm{~d}, 2 \mathrm{H}, J=8.5 \mathrm{~Hz}), 7.64(\mathrm{~d}, 2 \mathrm{H}, J=$ $8.5 \mathrm{~Hz}), 7.55(\mathrm{~d}, 2 \mathrm{H}, J=8.0 \mathrm{~Hz}), 7.45(\mathrm{~d}, 2 \mathrm{H}, J=8.0 \mathrm{~Hz}), 4.75(\mathrm{~d}, 2 \mathrm{H}, J=3.0 \mathrm{~Hz})$, $1.75(\mathrm{~s}, 1 \mathrm{H}) .{ }^{13} \mathrm{C} \mathrm{NMR}\left(126 \mathrm{MHz}, \mathrm{CDCl}_{3}\right) \delta 145.5,141.6,138.7,132.8,127.9,127.8$, 127.6, 119.1, 111.1, 65.0. FT-IR (KBr): 3480, 2230, 1637, 1495, 1384, 1032, $808 \mathrm{~cm}^{-1}$. ESI-MS: $m / z$ calcd for $\mathrm{C}_{14} \mathrm{H}_{12} \mathrm{NO}\left[\mathrm{M}+\mathrm{H}^{+}\right] 210.08$, found 210.08 .<smiles>OCc1ccc(C(=C(c2ccccc2)c2ccccc2)c2ccccc2)cc1</smiles>

\section{4-(Triphenylethenyl)-benzenemethanol (19)}

The desired compound was prepared with potassium 4-(2hydroxymethyl)phenyltrifluoroborate $(21.4 \mathrm{mg}, 0.1 \mathrm{mmol})$ and bromotriphenylethylene (33.2 $\mathrm{mg}, 0.1 \mathrm{mmol}$ ) using the general procedure (31.2 $\mathrm{mg}$, a white solid, $86 \%$ yield). $\mathrm{mp}=105{ }^{\circ} \mathrm{C} .{ }^{1} \mathrm{H}$ NMR $\left(500 \mathrm{MHz}, \mathrm{CDCl}_{3}\right) \delta 7.06(\mathrm{~m}, 11 \mathrm{H}), 6.99(\mathrm{~m}, 8 \mathrm{H}), 4.57(\mathrm{~s}, 2 \mathrm{H})$. ${ }^{13} \mathrm{C}$ NMR $\left(126 \mathrm{MHz}, \mathrm{CDCl}_{3}\right) \delta 143.9,143.4,141.3,140.7,139.1,131.7,131.5,127.9$, 127.8, 126.6, 126.5, 65.4. FT-IR (KBr): 3418, 3021, 2923, 1637, 1491, 1442, 1029, 908 , $763,698 \mathrm{~cm}^{-1}$. ESI-MS: $\mathrm{m} / z$ calcd for $\mathrm{C}_{27} \mathrm{H}_{21} \mathrm{O}\left[\mathrm{M}-\mathrm{H}^{+}\right] 361.15$, found 361.15 . 


\section{References}

1. Ondruschka, B.; Bonrath, W.; Stuerga, D. In Microwaves in Organic Synthesis; Loupy, A., Eds.; Wiley-VCH: Weinheim, 2006; Vol. 1; Chapter 2.

2. Smoum, R.; Rubinstein, A.; Srebnik, M. Org. Biomol. Chem. 2005, 3, 941.

3. Arvela R. K.; Leadbeater, N. E.; Mack, T. L.; Kormos, C. M. Tetrahedron Lett. 2006, 47, 217.

4. Molander, G. A.; Biolatto, B. J. Org. Chem. 2003, 68, 4302. 

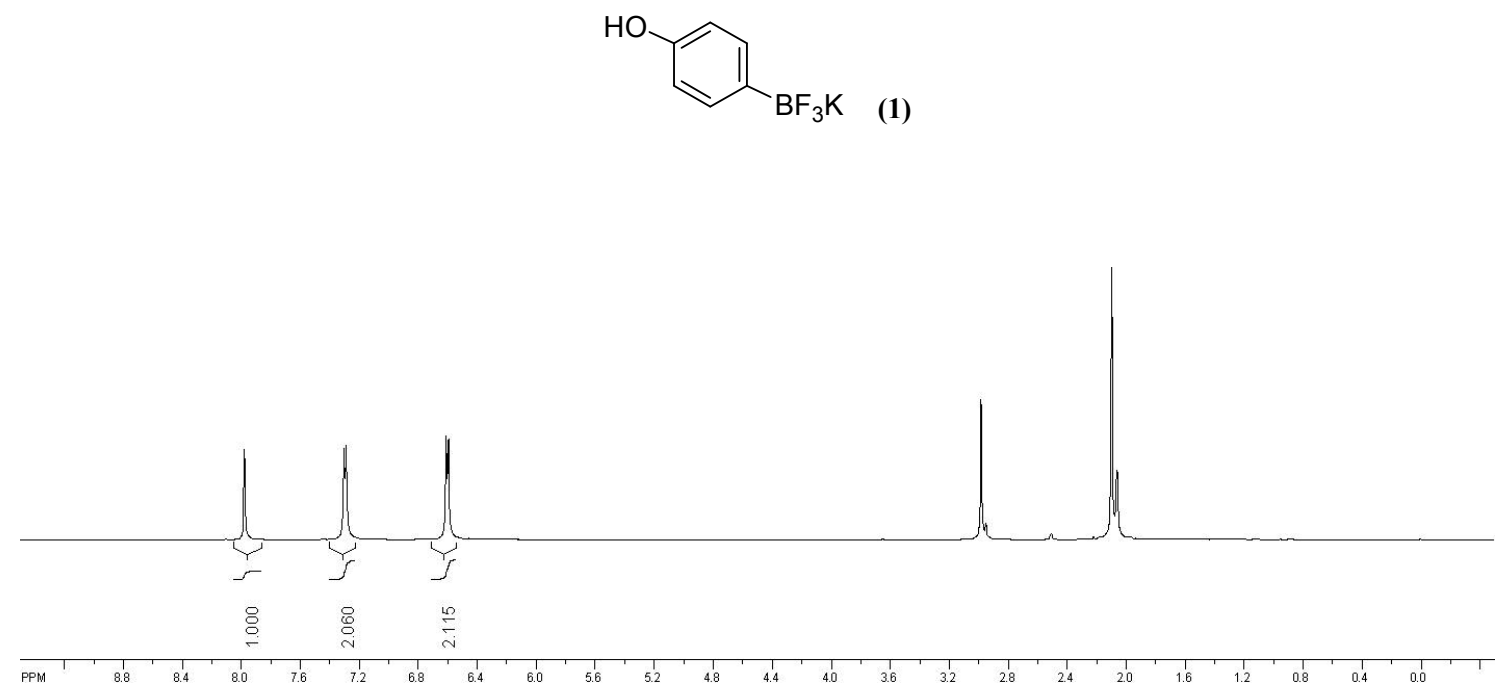
$500 \mathrm{MHz}{ }^{1} \mathrm{H}$ NMR of Compound (1) in Acetone- $d_{6}+$ DMSO- $d_{6}$
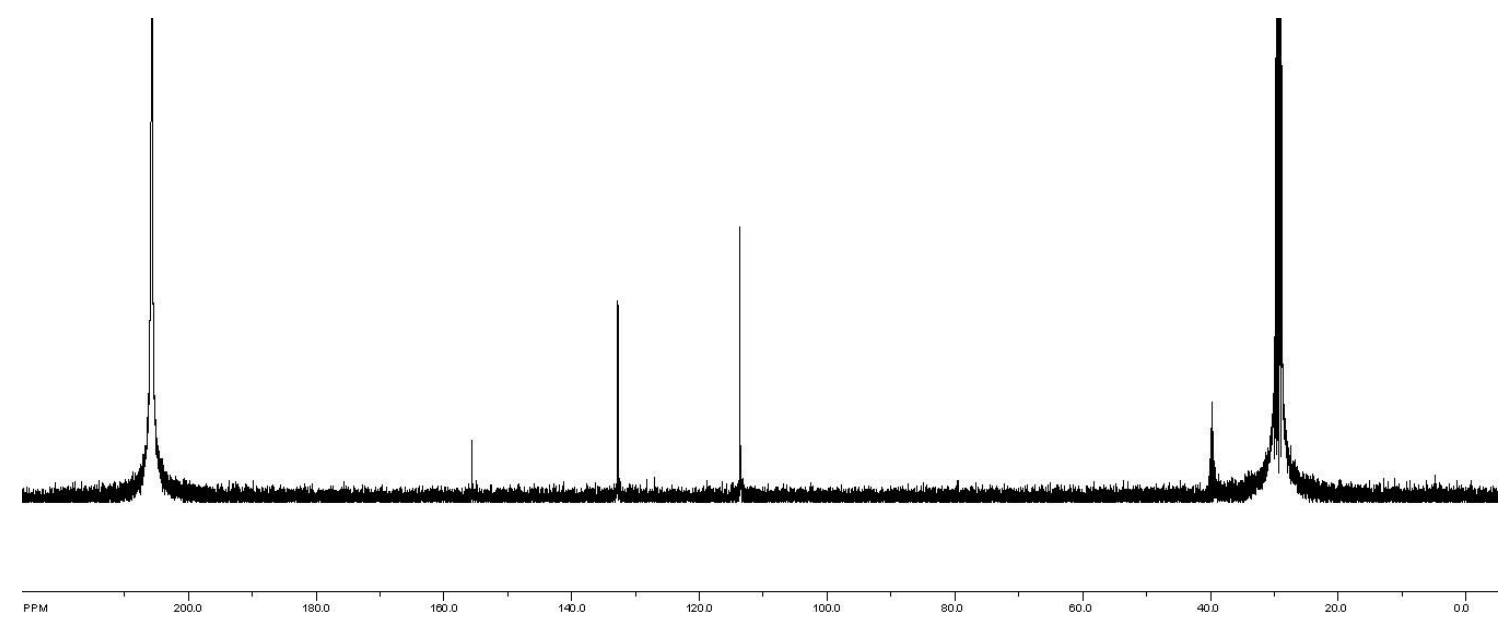

$126 \mathrm{MHz}{ }^{13} \mathrm{C}$ NMR of Compound (1) in Acetone- $d_{6}+$ DMSO- $d_{6}$

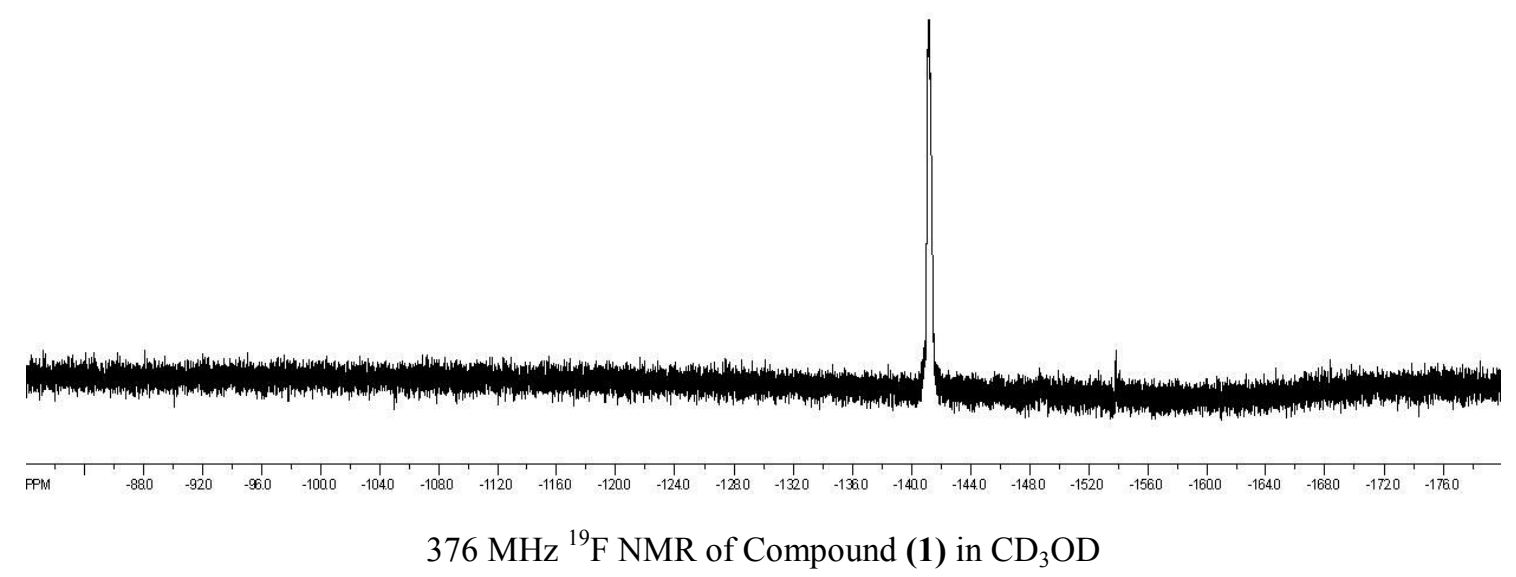



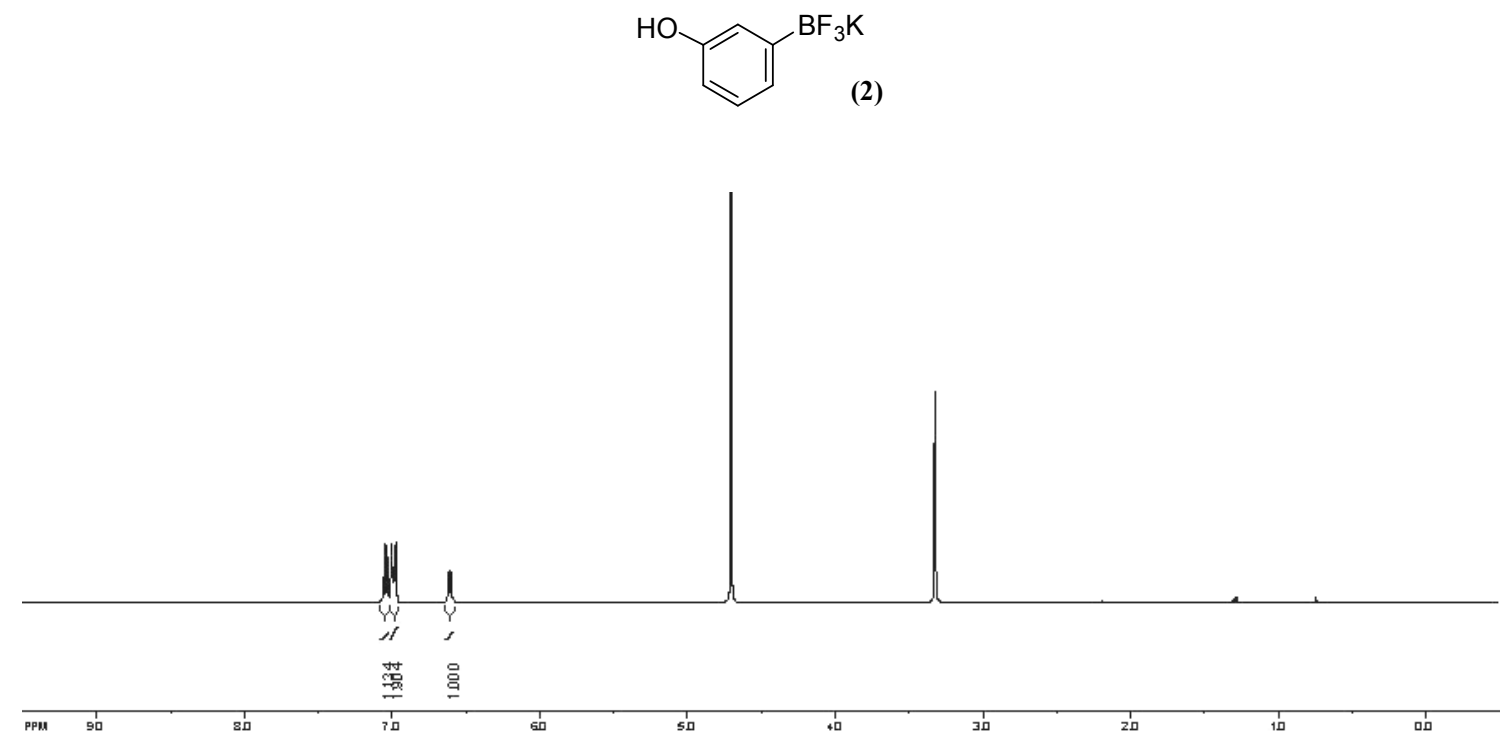

$500 \mathrm{MHz}{ }^{1} \mathrm{H}$ NMR of Compound (2) in $\mathrm{CD}_{3} \mathrm{OD}$

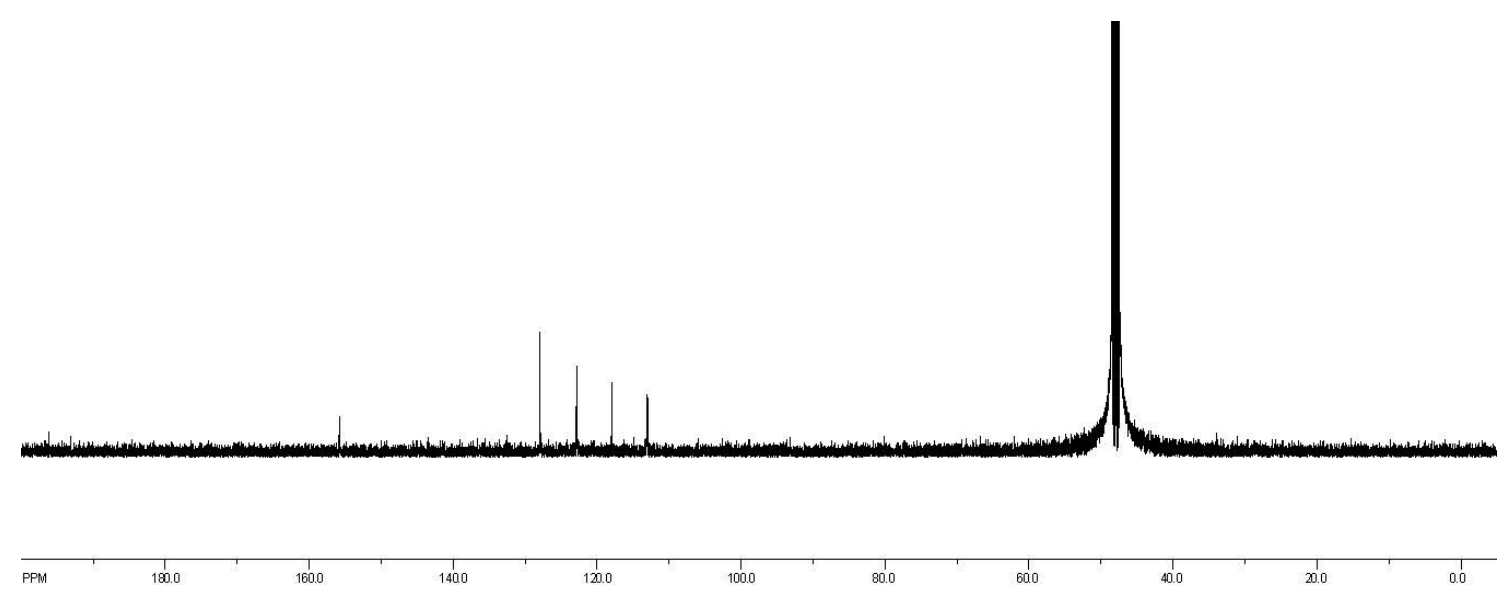

$126 \mathrm{MHz}{ }^{13} \mathrm{C}$ NMR of Compound (2) in $\mathrm{CD}_{3} \mathrm{OD}$

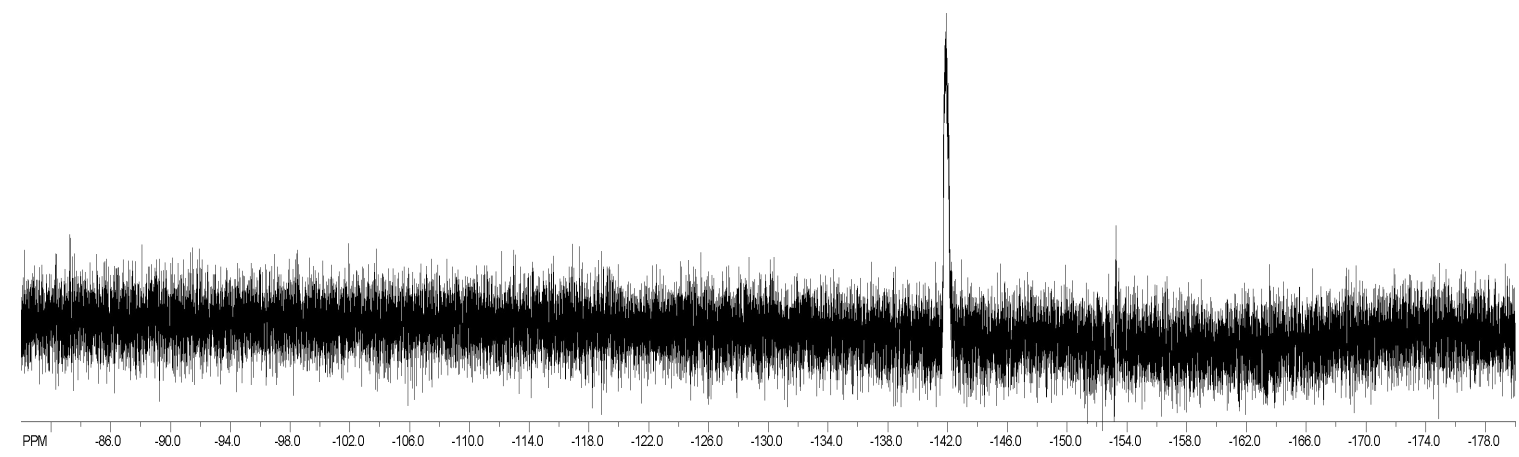

$376 \mathrm{MHz}{ }^{19} \mathrm{~F}$ NMR of Compound (2) in $\mathrm{CD}_{3} \mathrm{OD}$ 

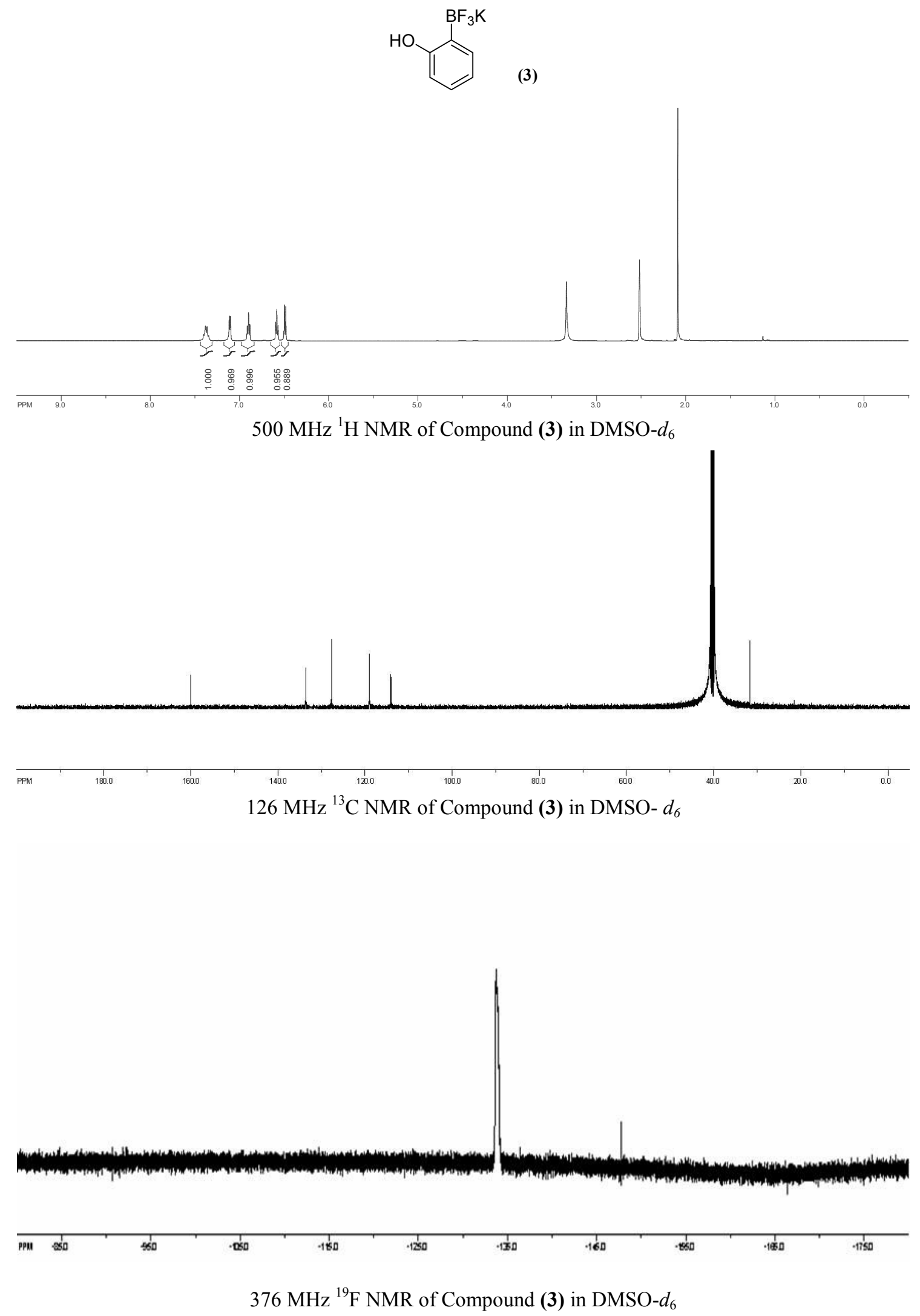


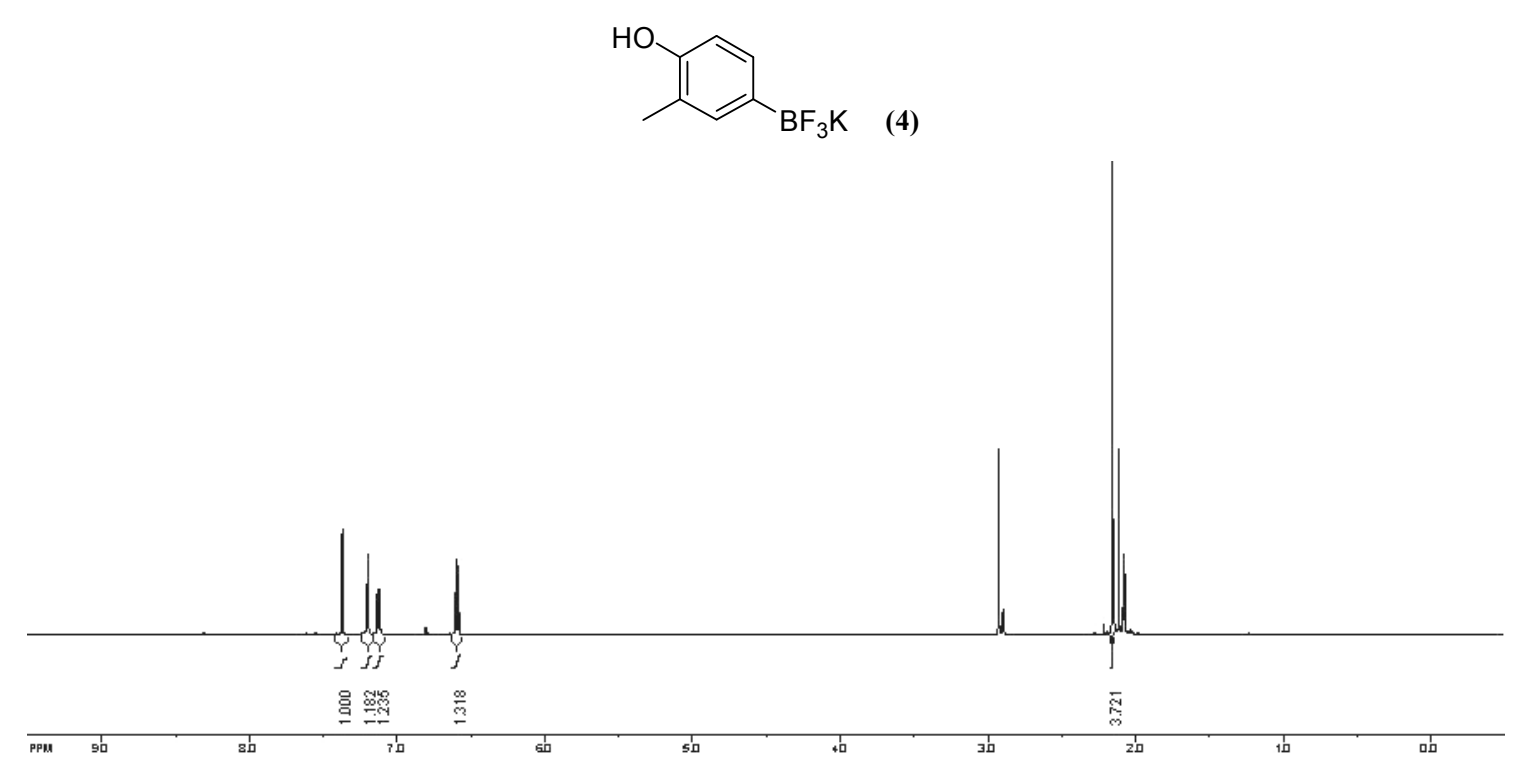

$500 \mathrm{MHz}{ }^{1} \mathrm{H}$ NMR of Compound (4) in Acetone- $d_{6}$
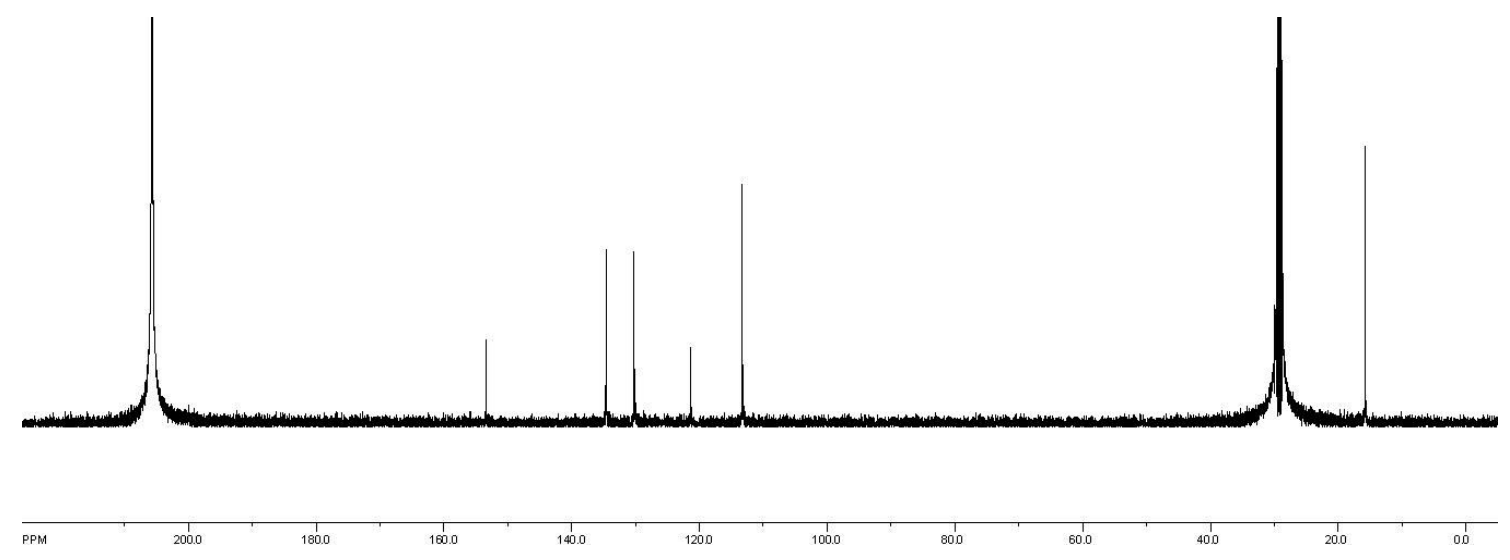

$126 \mathrm{MHz}{ }^{13} \mathrm{C}$ NMR of Compound (4) in Acetone- $d_{6}$

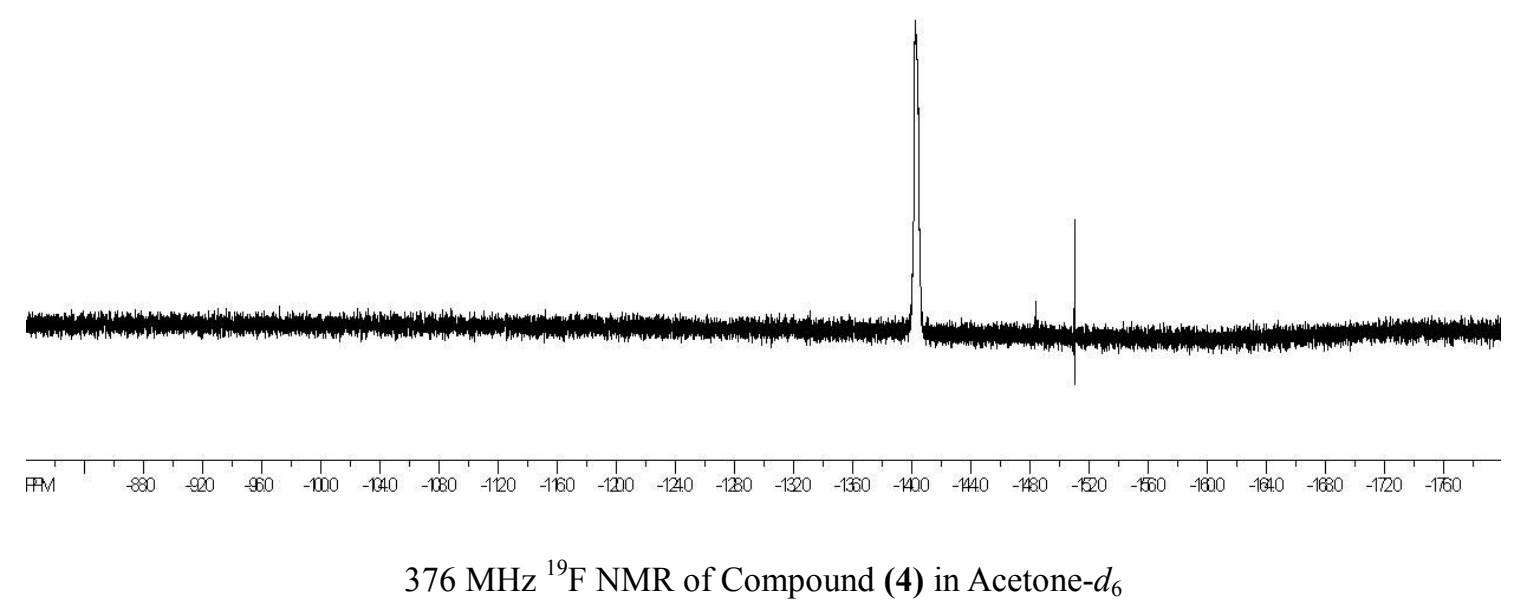



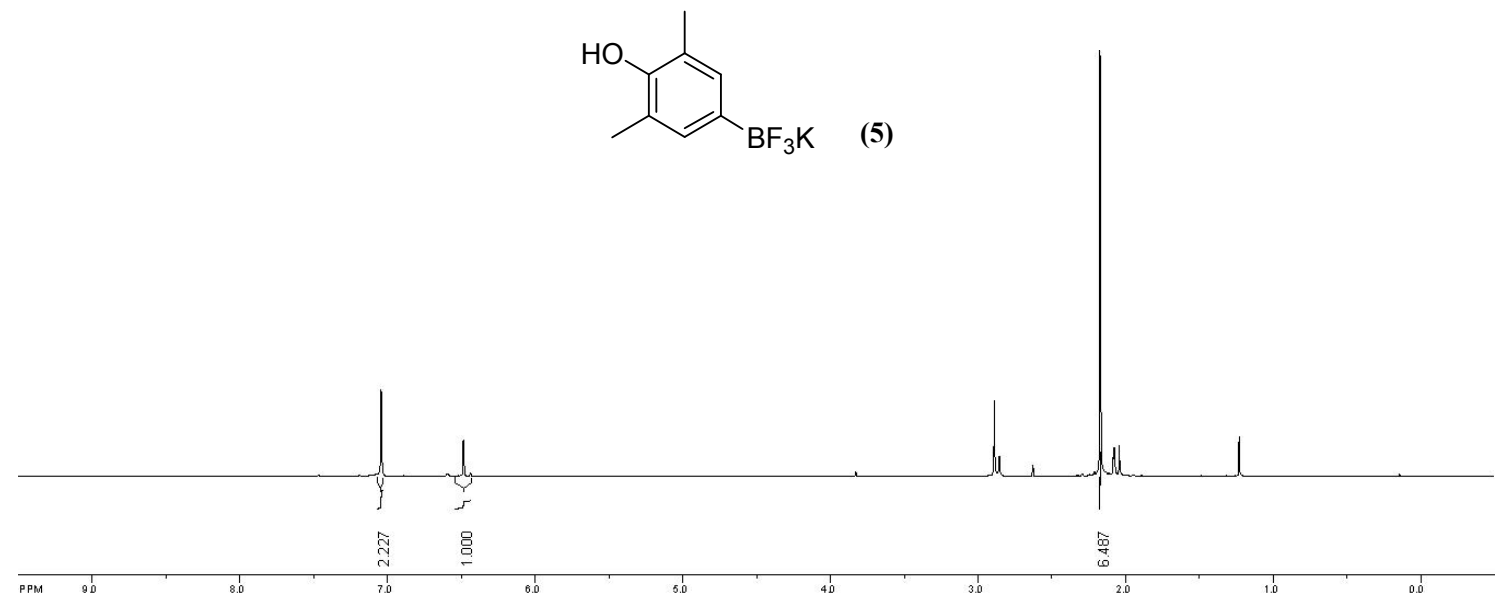

$500 \mathrm{MHz}{ }^{1} \mathrm{H}$ NMR of Compound (5) in Acetone- $d_{6}$
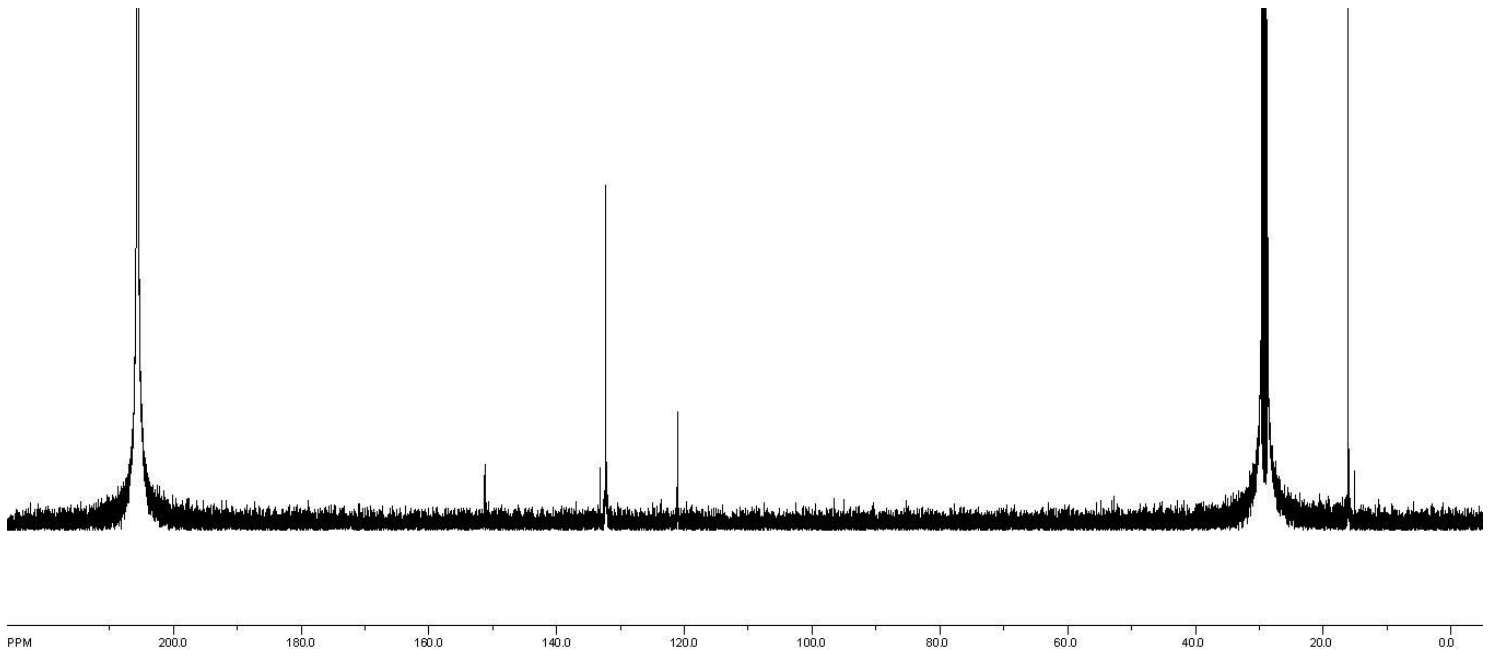

$126 \mathrm{MHz}{ }^{13} \mathrm{C}$ NMR of Compound (5) in Acetone- $d_{6}$

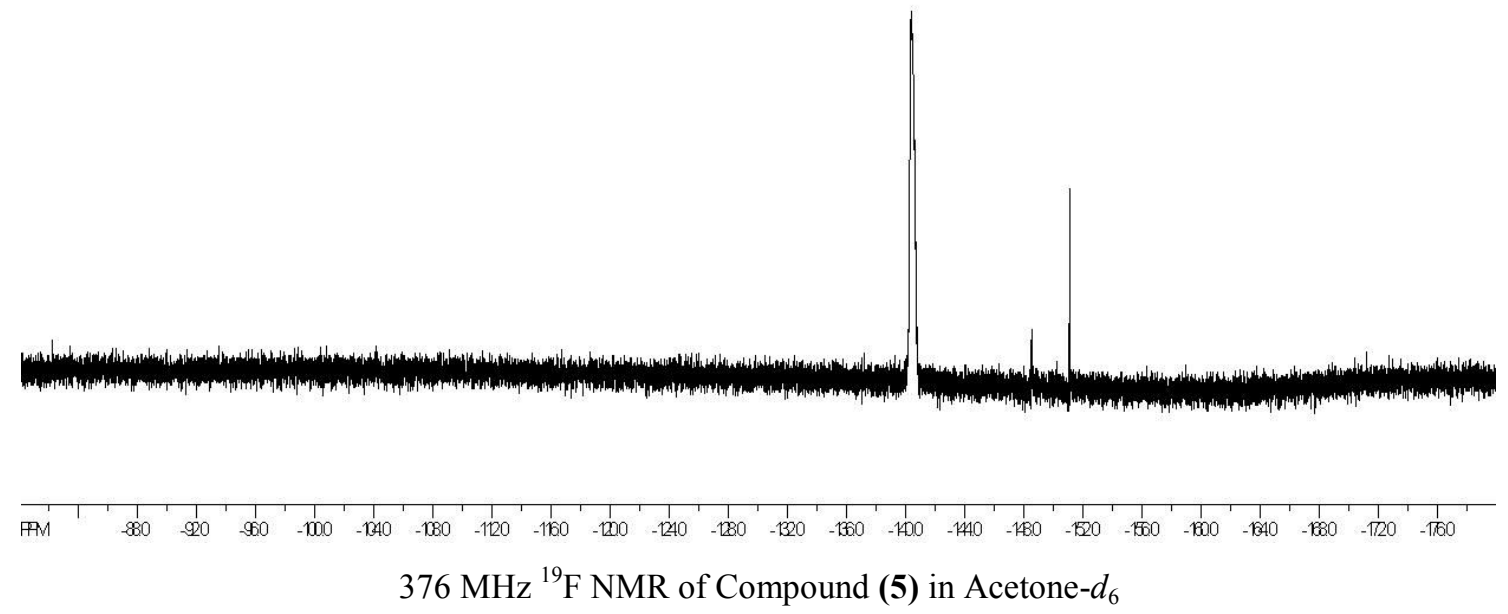




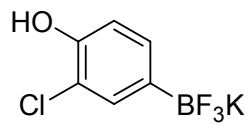

(6)

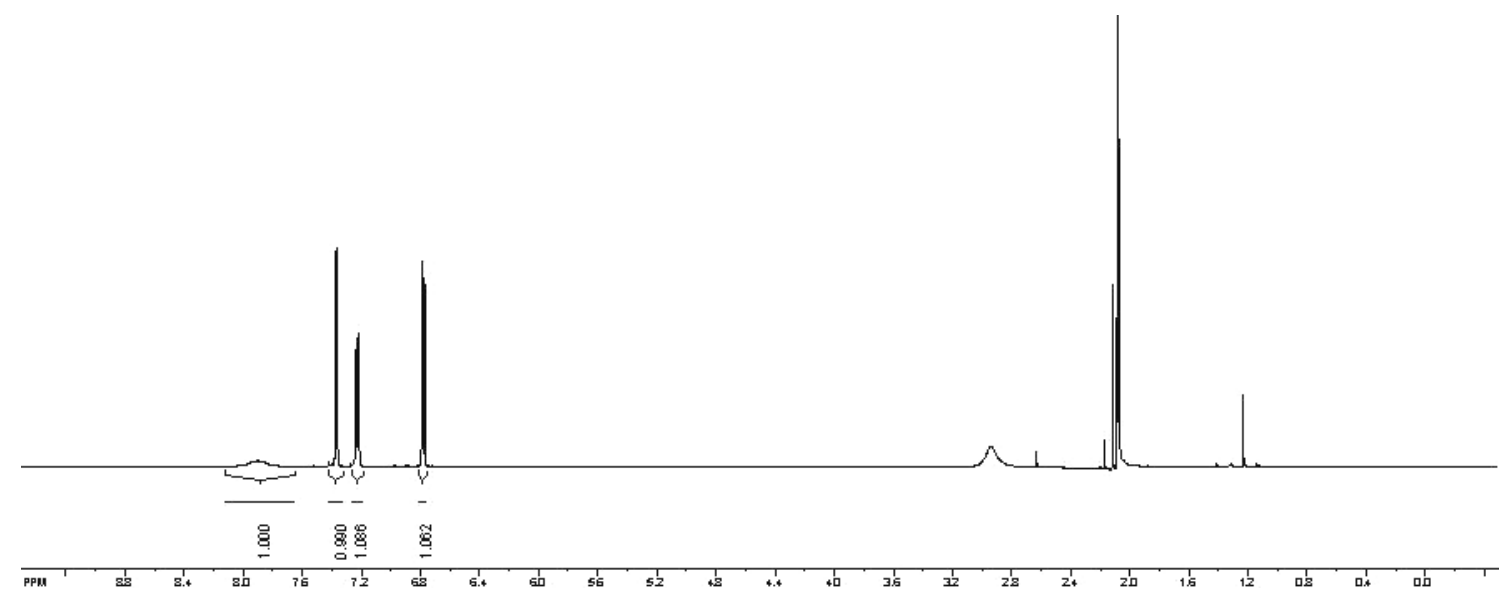

$500 \mathrm{MHz}{ }^{1} \mathrm{H}$ NMR of Compound (6) in Acetone- $d_{6}$
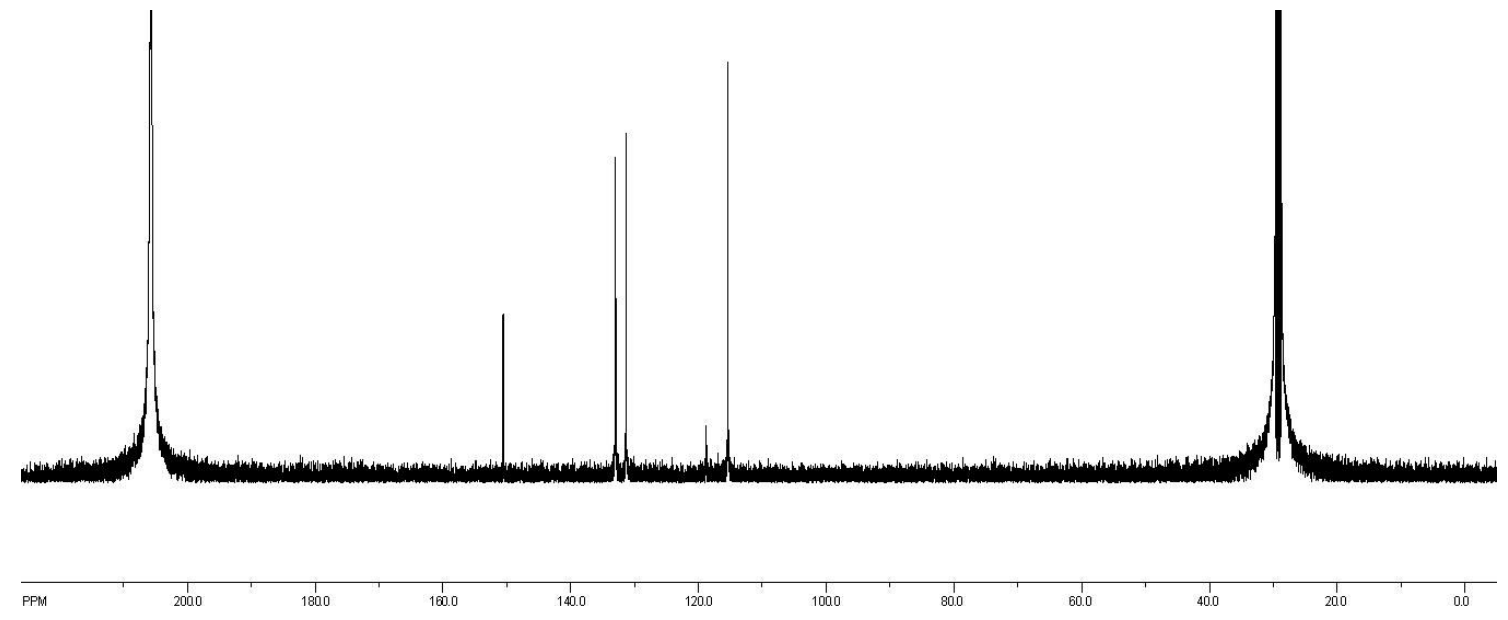

$126 \mathrm{MHz}{ }^{13} \mathrm{C}$ NMR of Compound (6) in Acetone- $d_{6}$

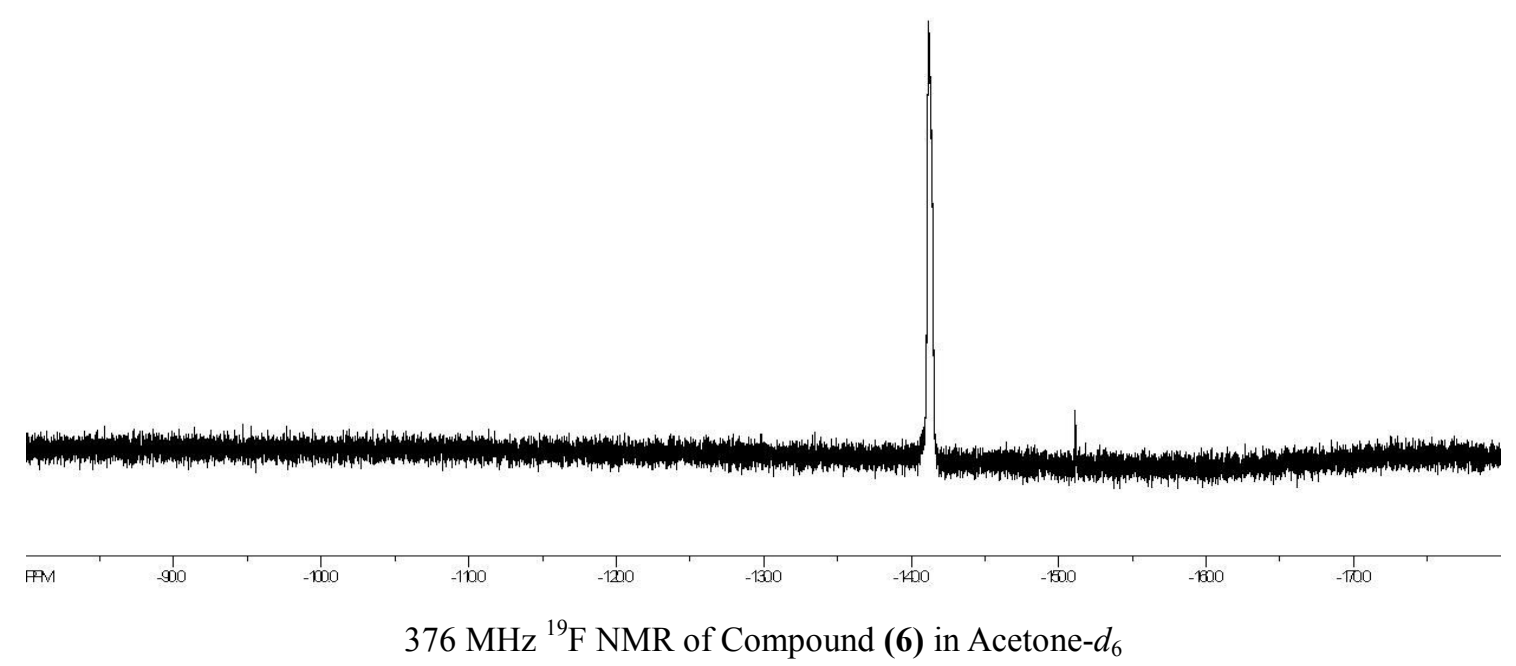



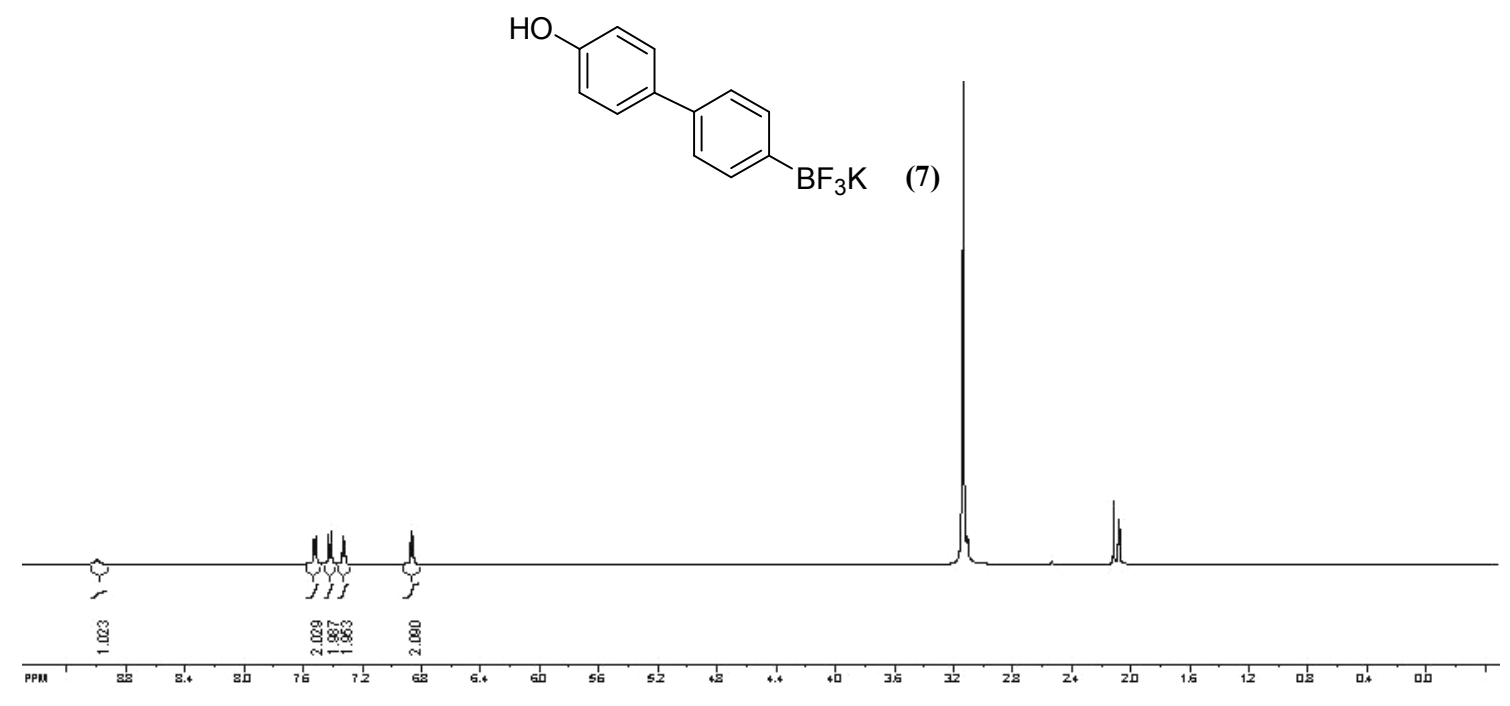

$500 \mathrm{MHz}{ }^{1} \mathrm{H}$ NMR of Compound (7) in Acetone- $d_{6}+$ DMSO- $d_{6}$
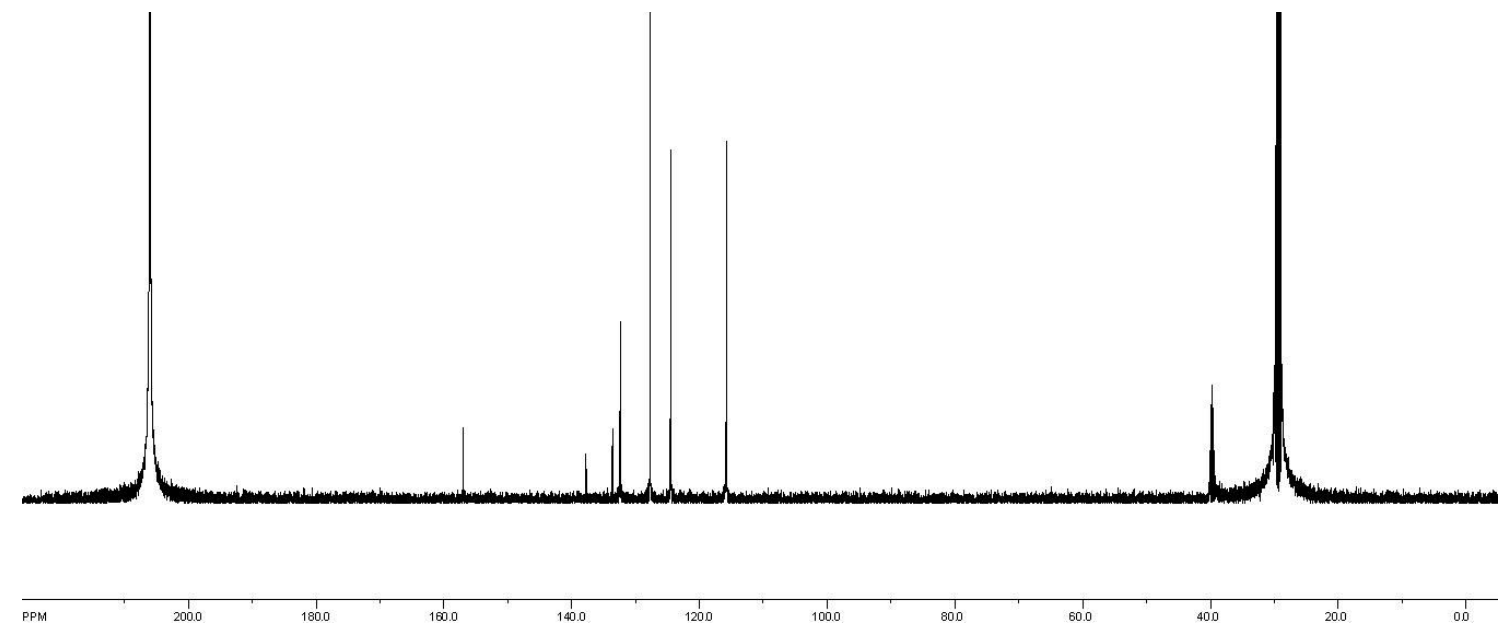

$126 \mathrm{MHz}{ }^{13} \mathrm{C}$ NMR of Compound (7) in Acetone- $d_{6}+$ DMSO- $d_{6}$

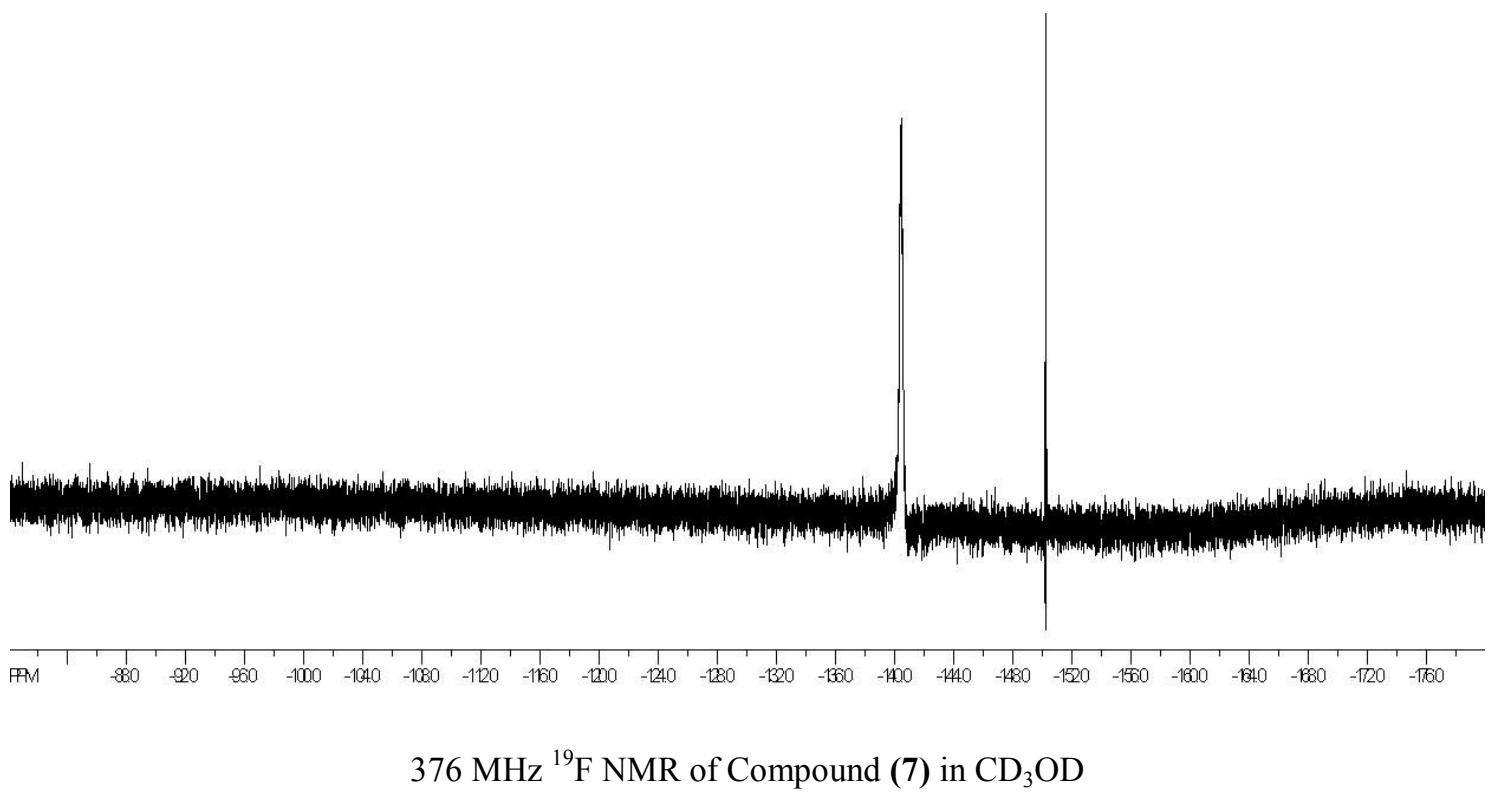


$\mathrm{HO}$<smiles>[B-]c1ccc2ccc(C)cc2c1</smiles>

(8)

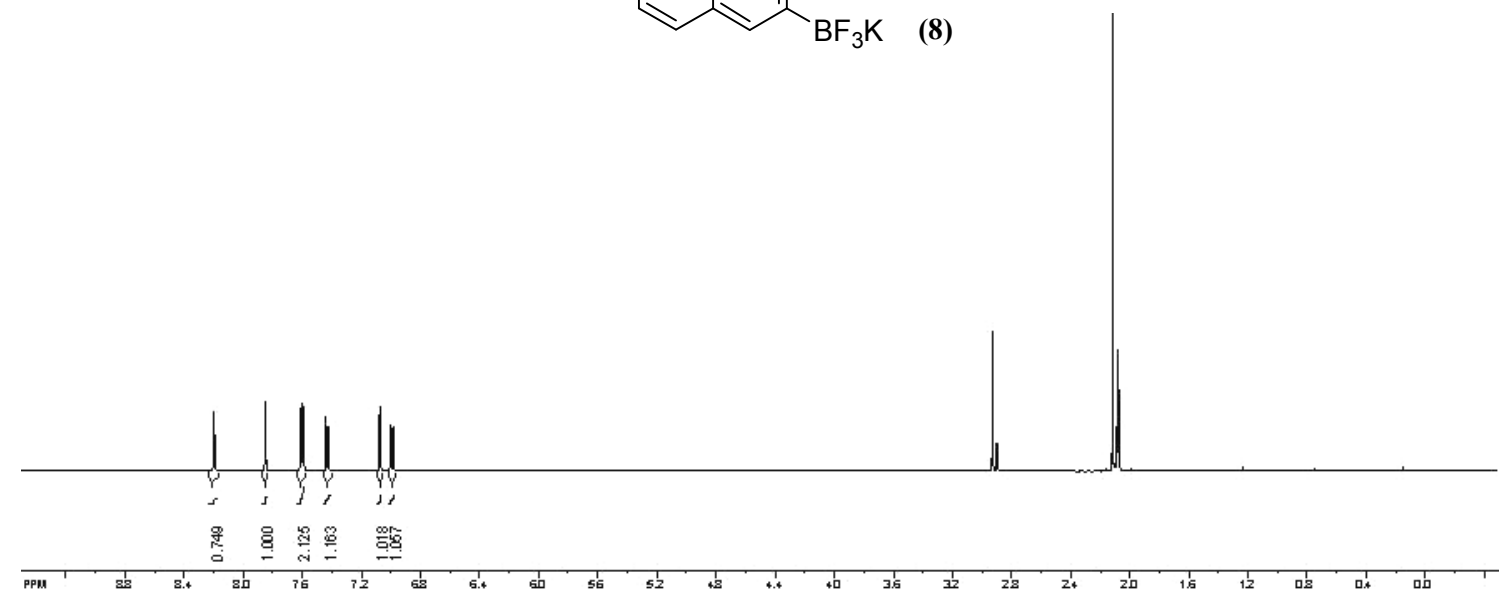

$500 \mathrm{MHz}{ }^{1} \mathrm{H}$ NMR of Compound (8) in Acetone- $d_{6}$
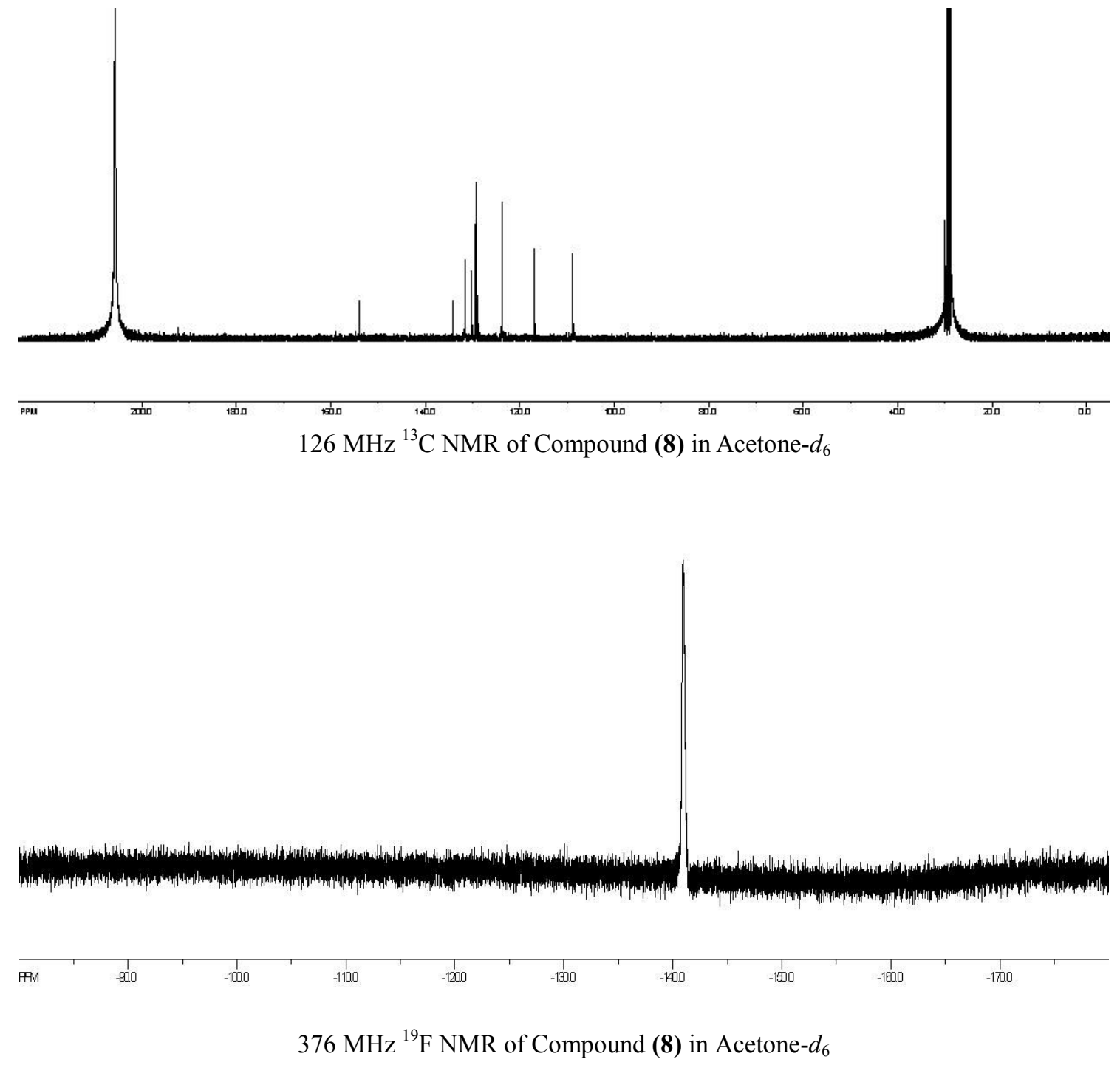


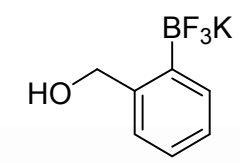

(9)

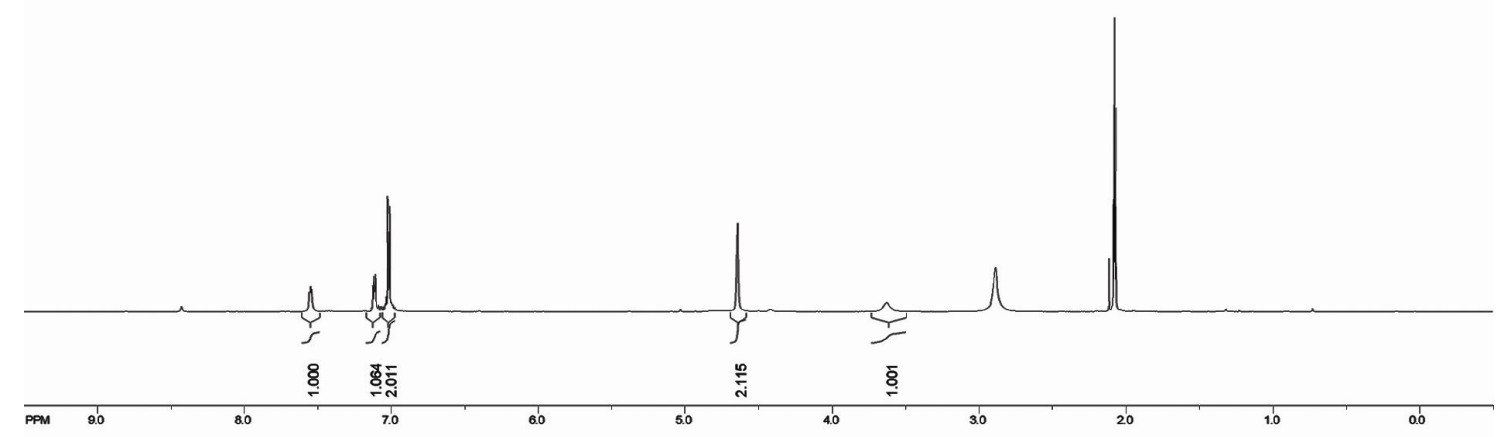

$500 \mathrm{MHz}{ }^{1} \mathrm{H}$ NMR of Compound (9) in Acetone- $d_{6}$
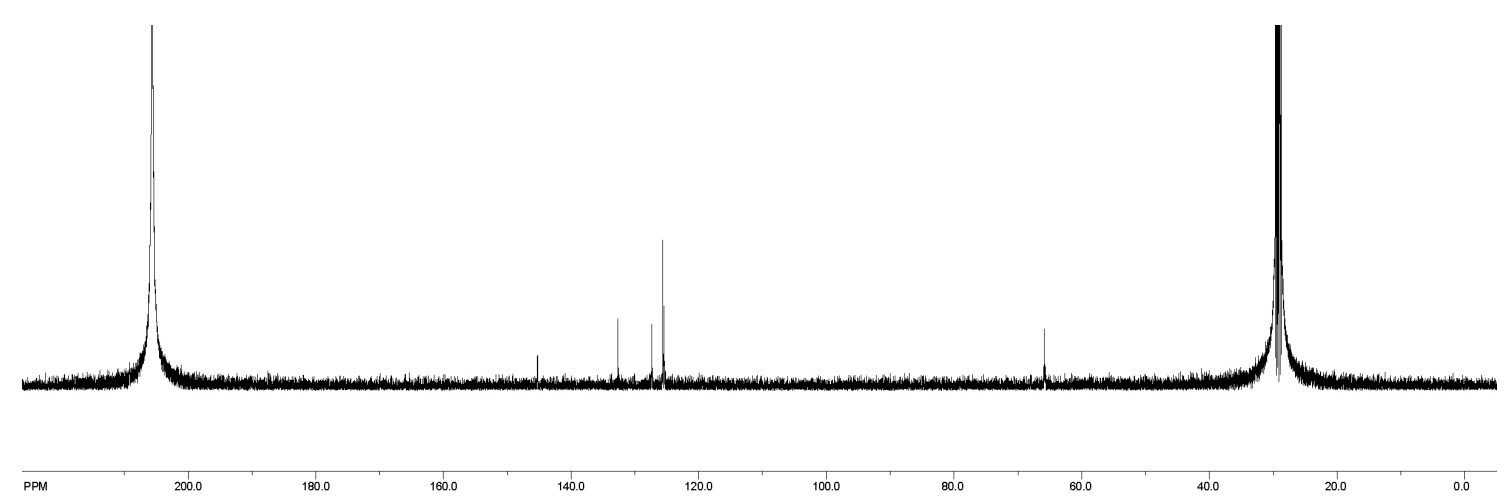

$126 \mathrm{MHz}{ }^{13} \mathrm{C}$ NMR of Compound (9) in Acetone- $d_{6}$

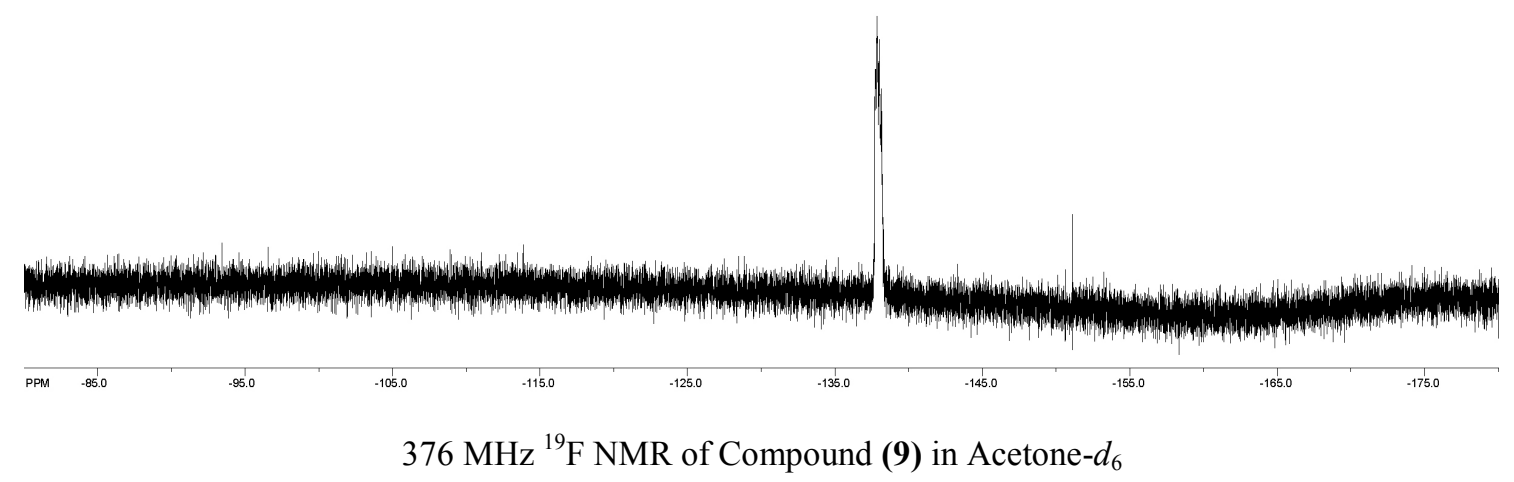




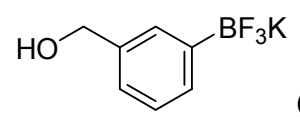

(10)

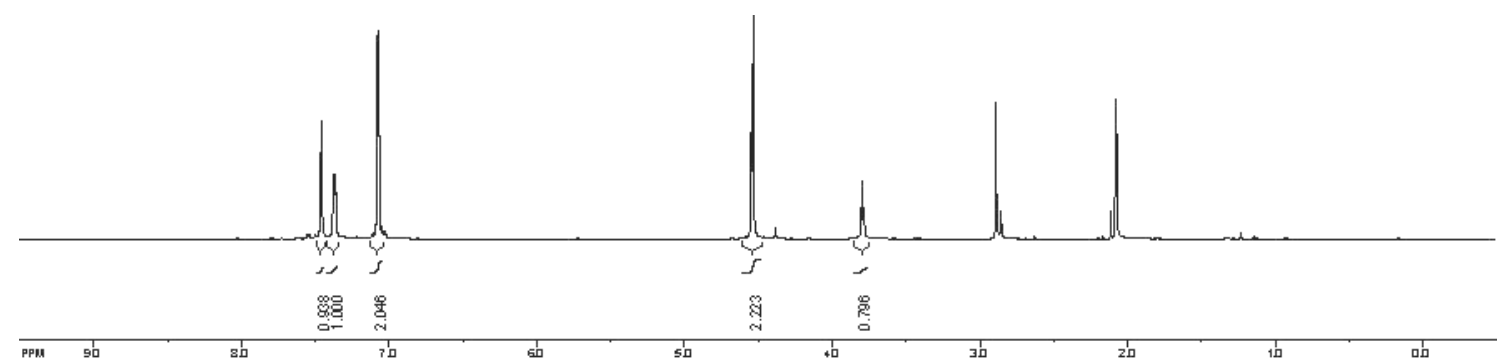

$500 \mathrm{MHz}{ }^{1} \mathrm{H}$ NMR of Compound (10) in Acetone- $d_{6}$
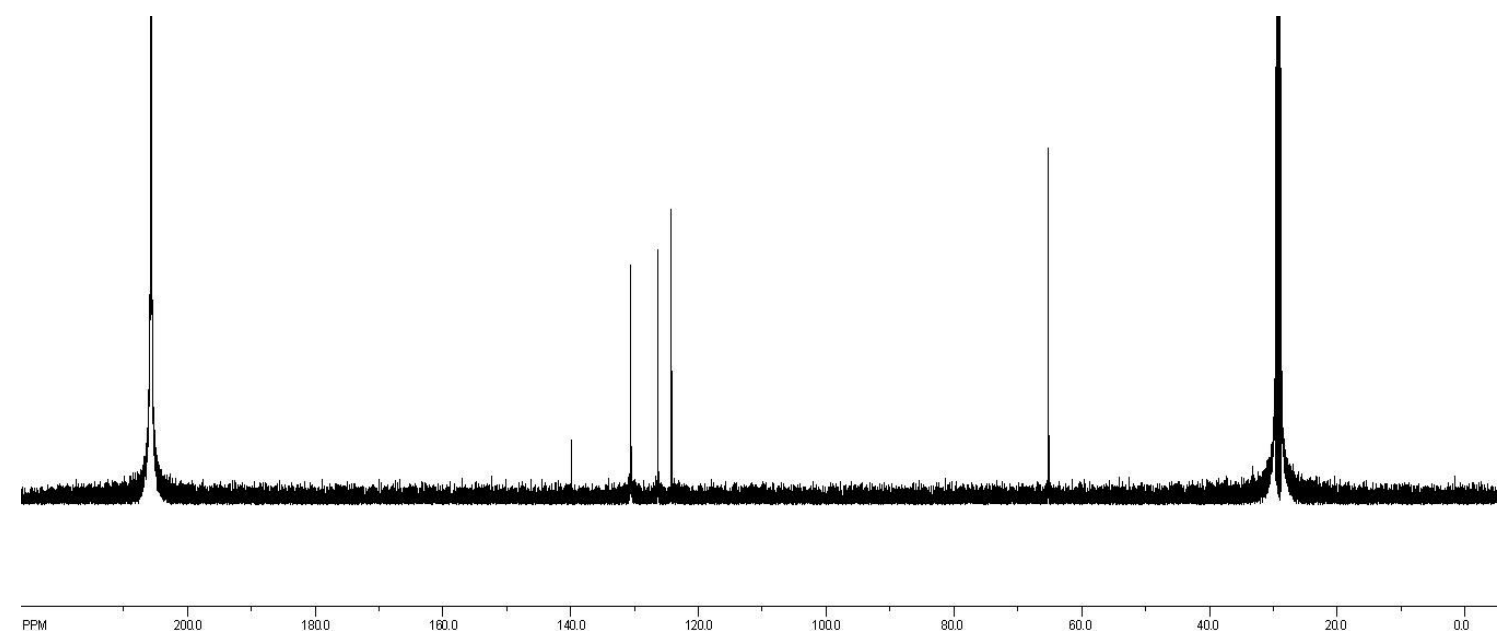

$126 \mathrm{MHz}{ }^{13} \mathrm{C}$ NMR of Compound (10) in Acetone- $d_{6}$

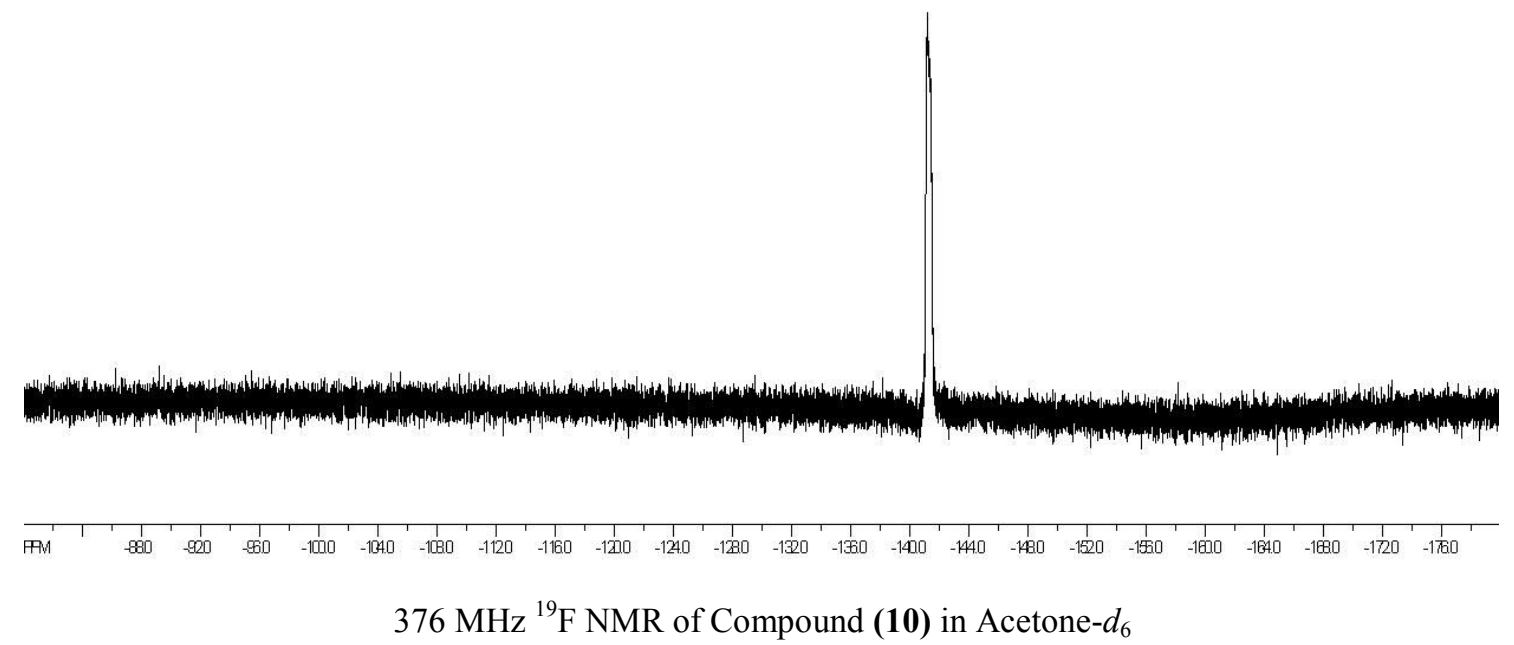



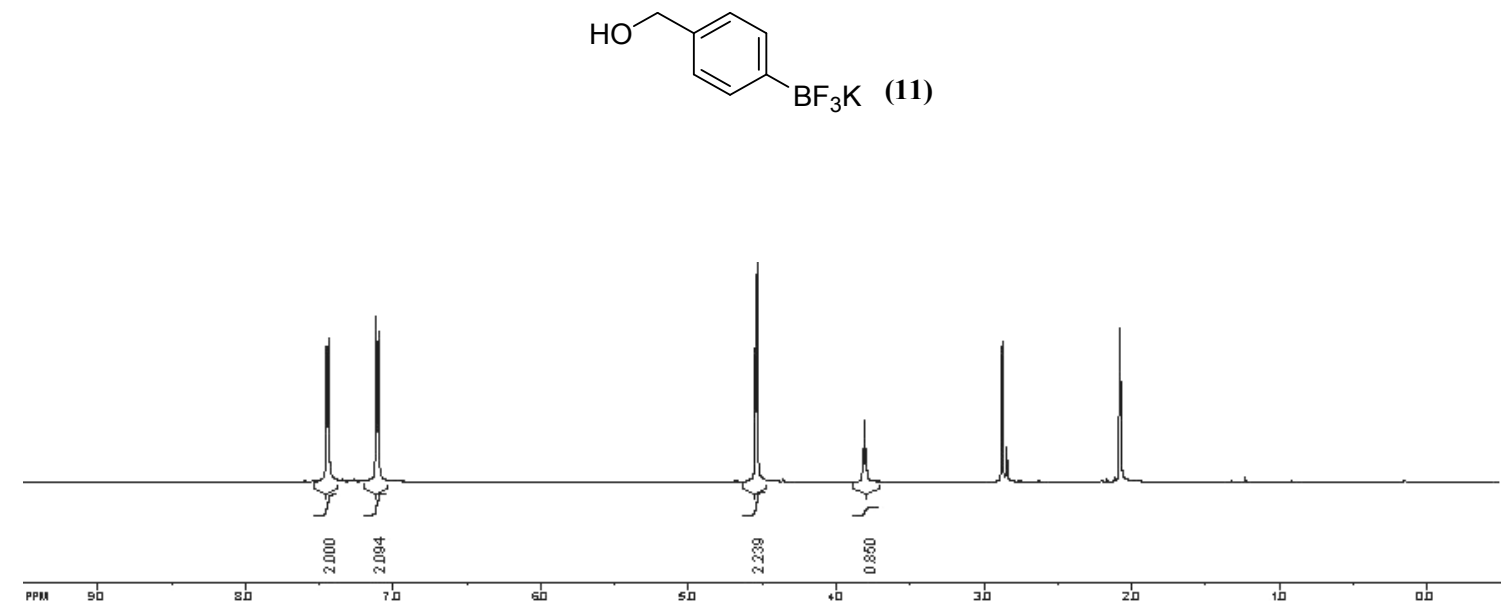

$500 \mathrm{MHz}{ }^{1} \mathrm{H}$ NMR of Compound (11) in Acetone- $d_{6}$
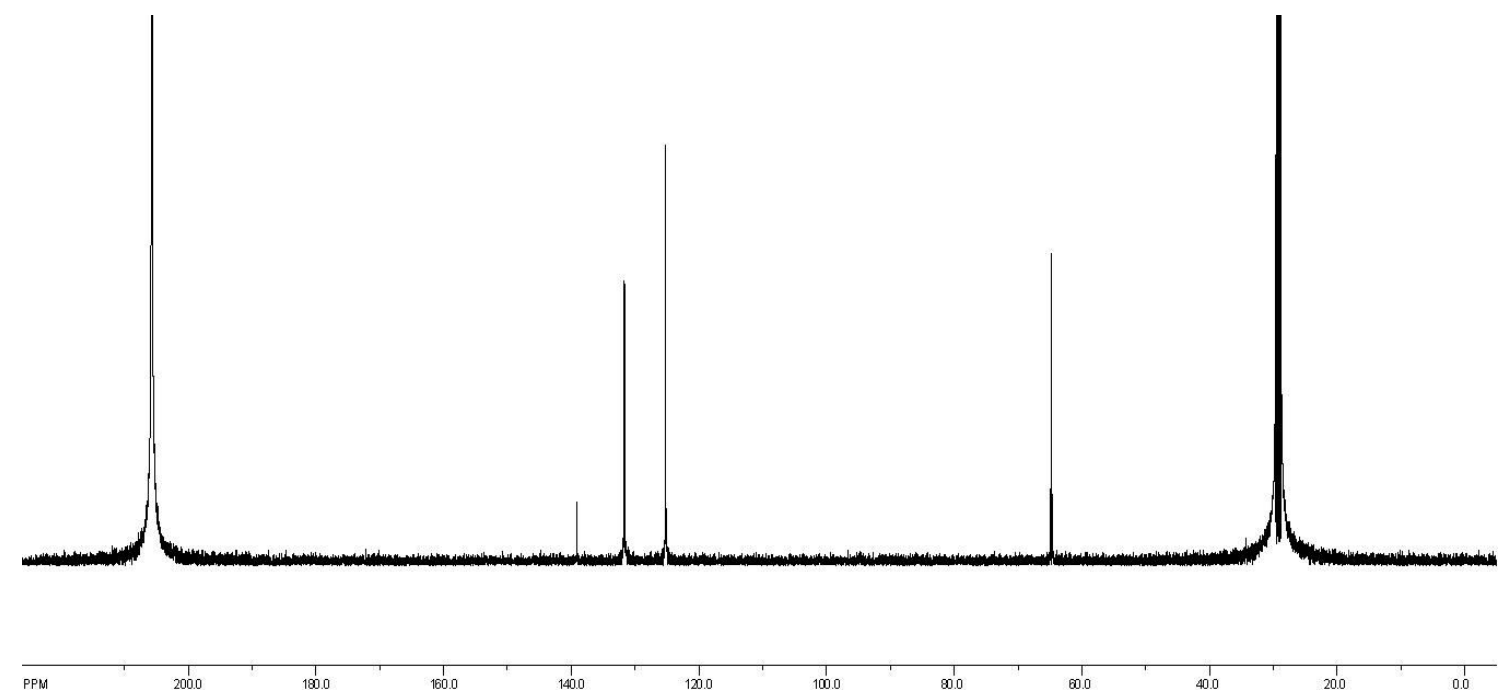

$126 \mathrm{MHz}{ }^{13} \mathrm{C}$ NMR of Compound (11) in Acetone- $d_{6}$

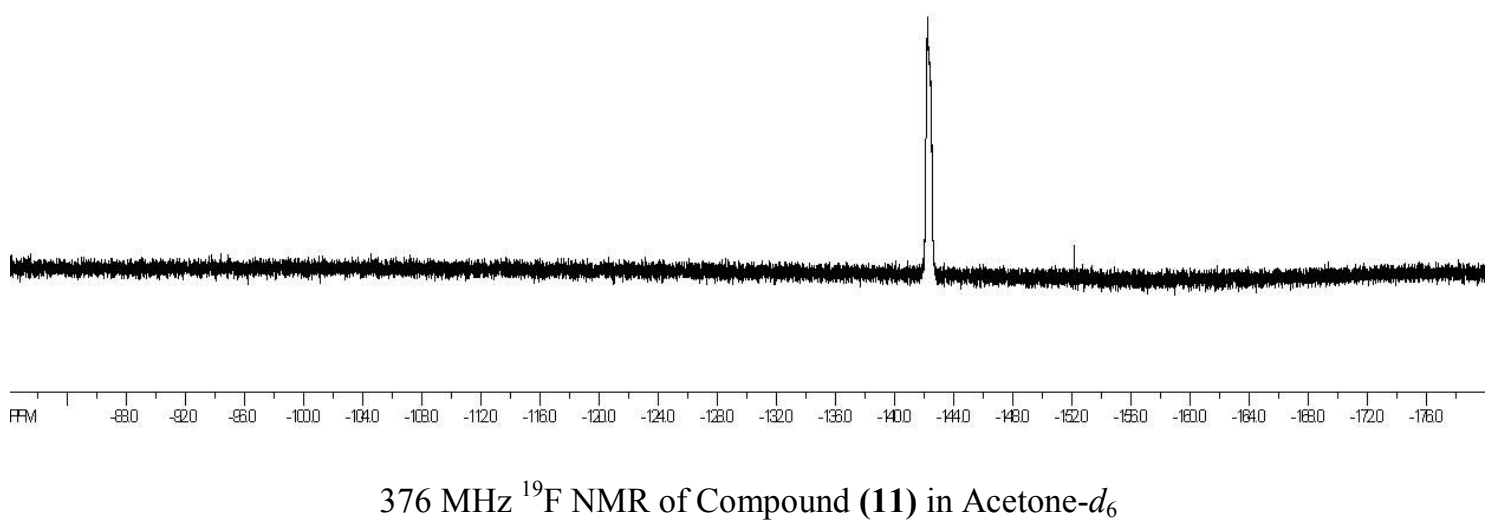



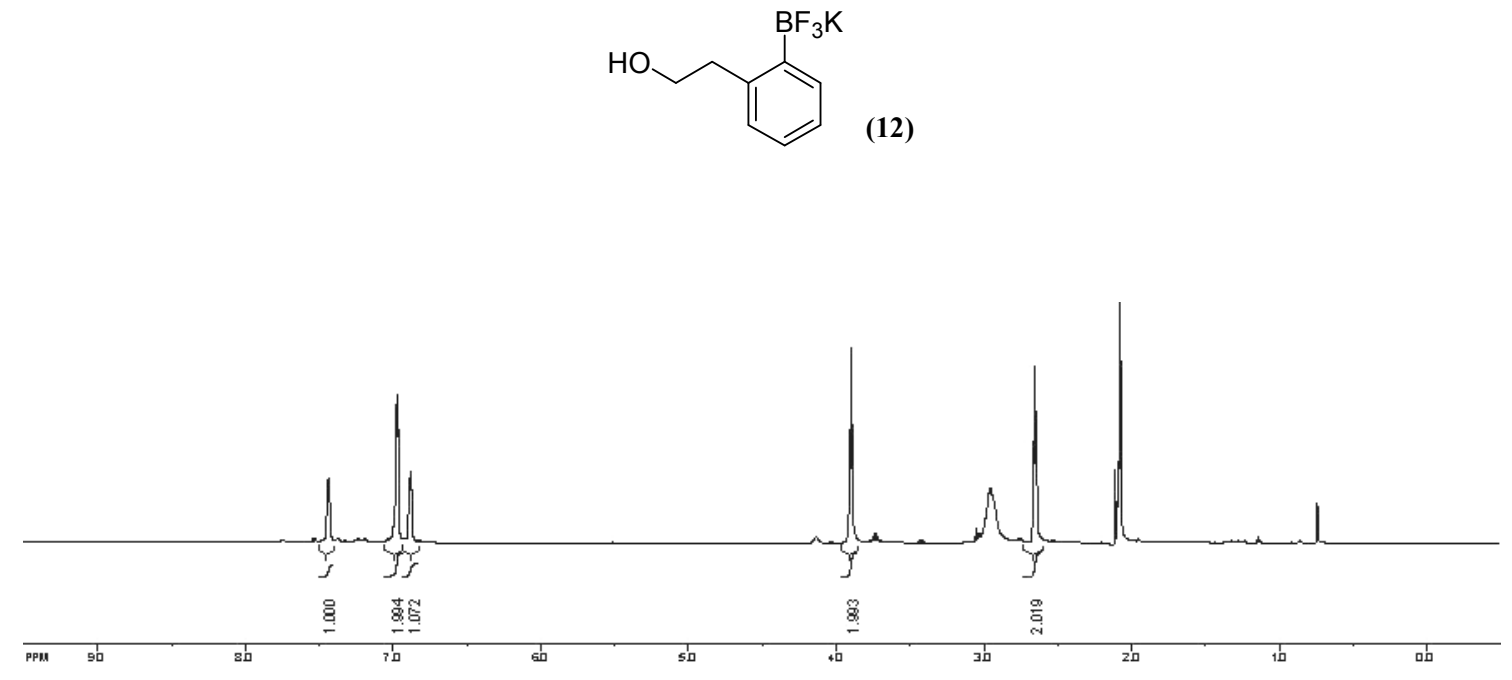

$500 \mathrm{MHz}{ }^{1} \mathrm{H}$ NMR of Compound (12) in Acetone- $d_{6}$
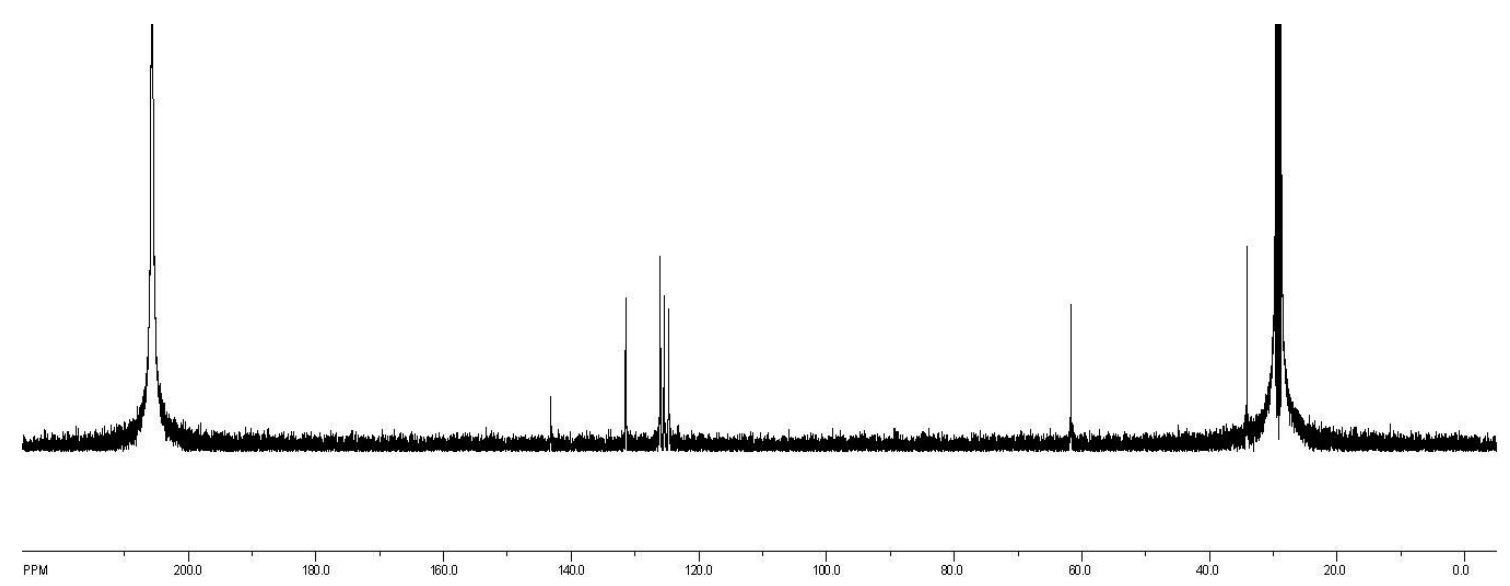

$126 \mathrm{MHz}{ }^{13} \mathrm{C}$ NMR of Compound (12) in Acetone- $d_{6}$

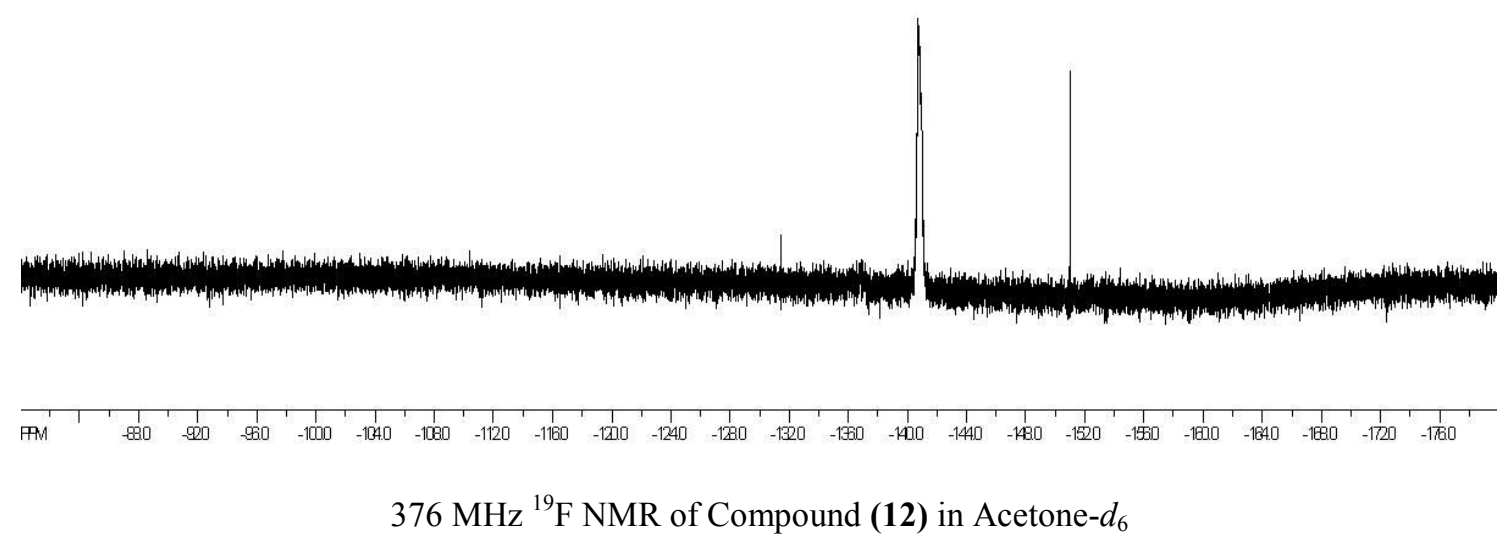


<smiles>OCCc1cccc(Br)c1</smiles>

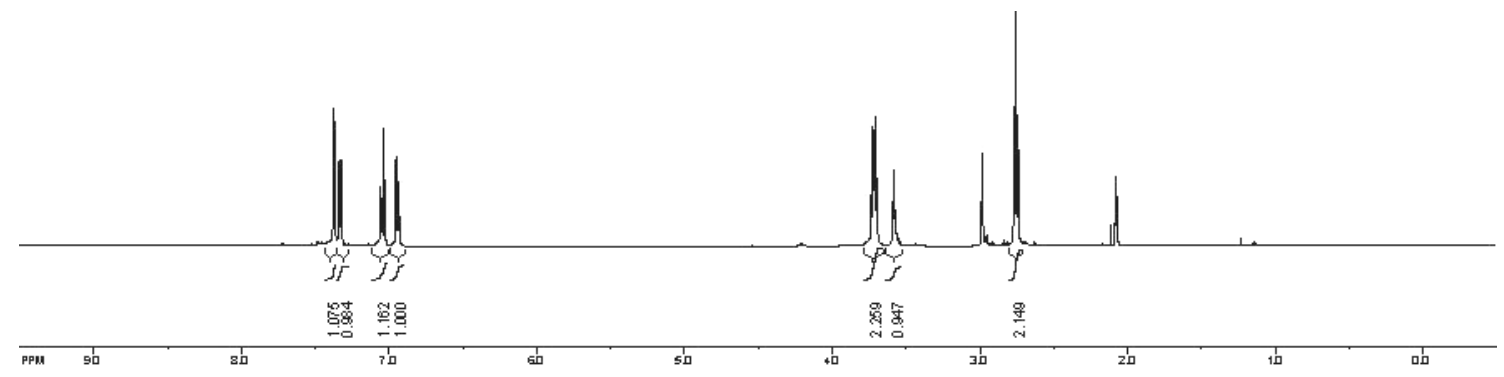

$500 \mathrm{MHz}{ }^{1} \mathrm{H}$ NMR of Compound (13) in Acetone- $d_{6}$
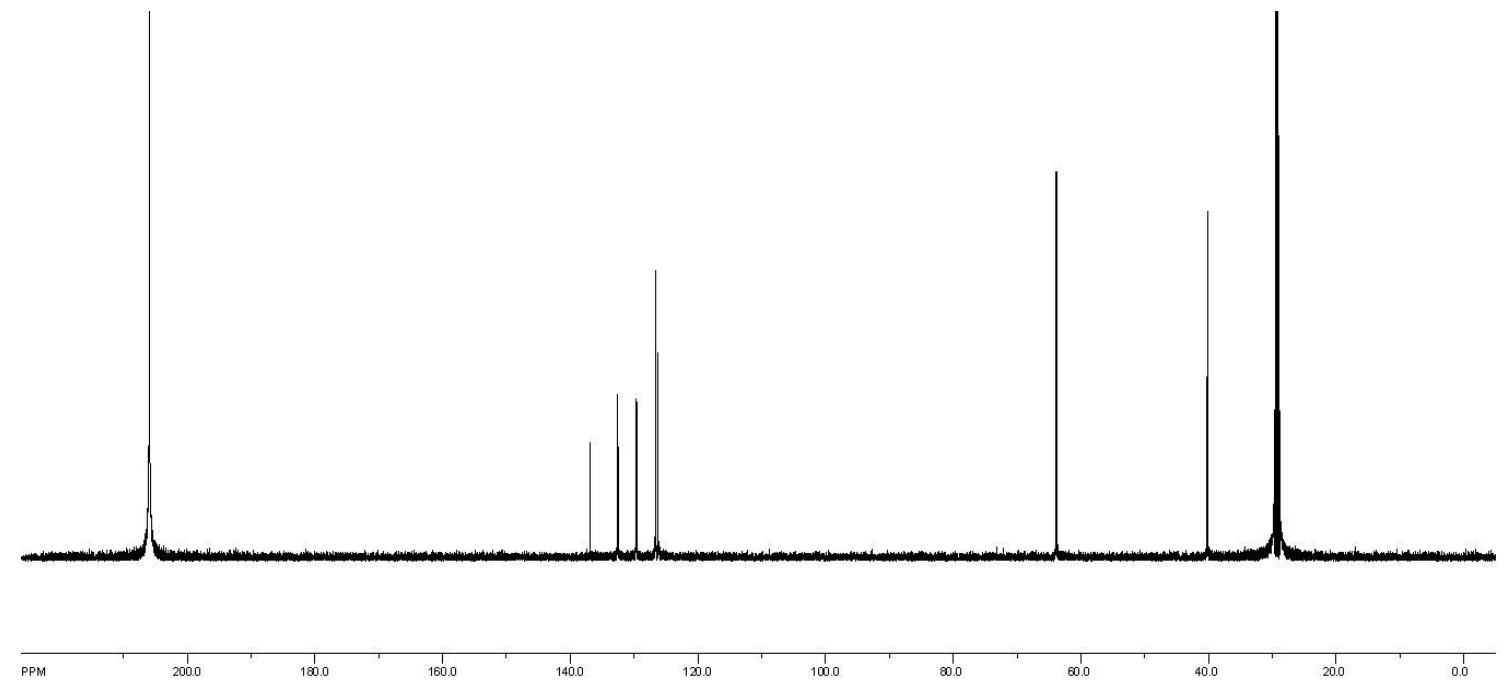

$126 \mathrm{MHz}{ }^{13} \mathrm{C}$ NMR of Compound (13) in Acetone- $d_{6}$

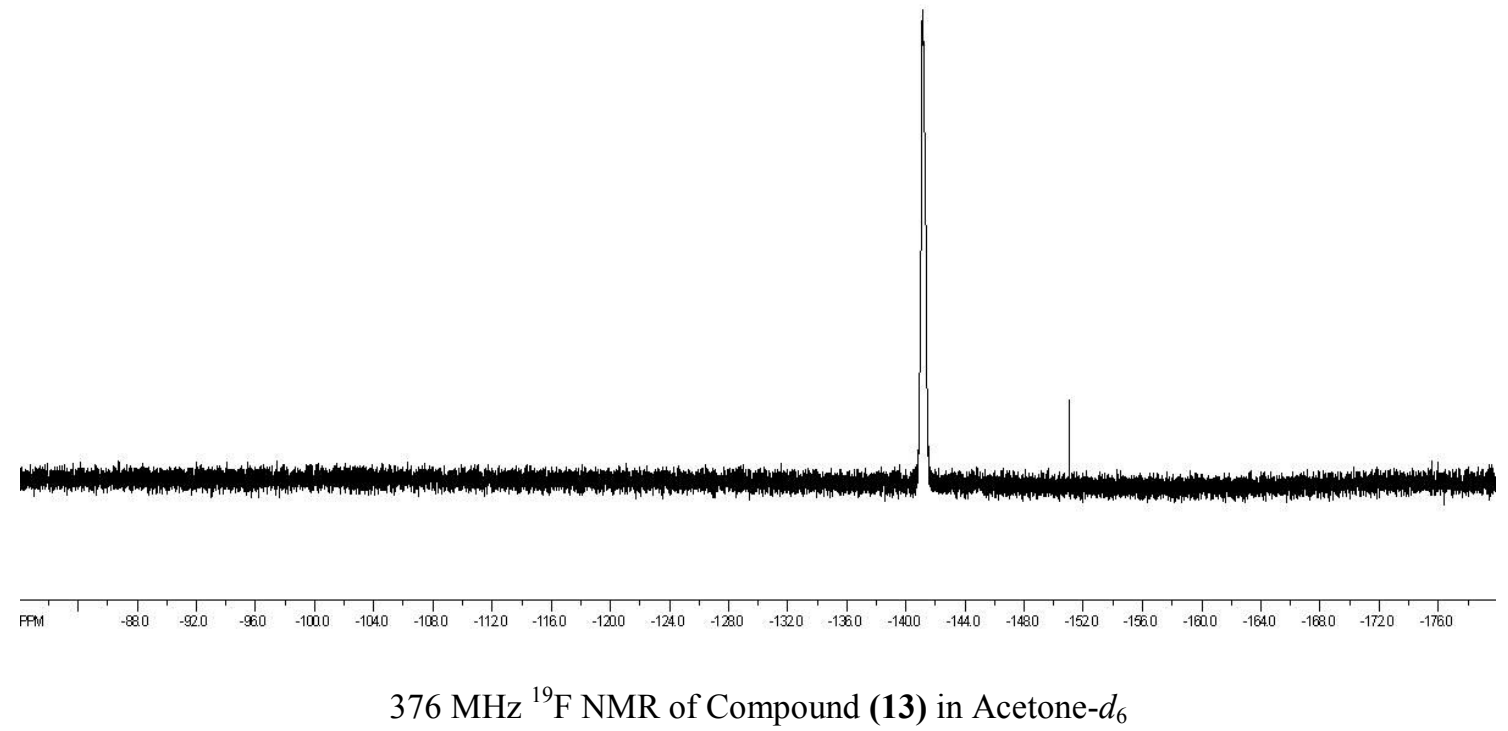



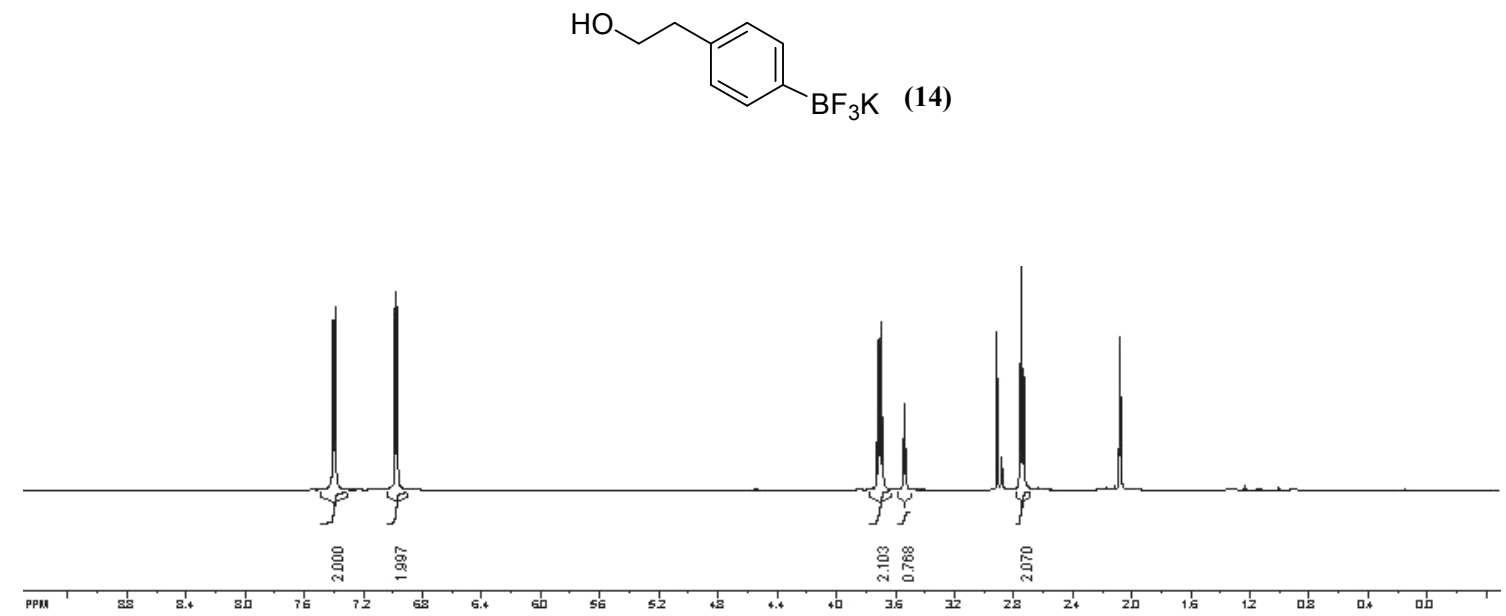

$500 \mathrm{MHz}{ }^{1} \mathrm{H}$ NMR of Compound (14) in Acetone- $d_{6}$
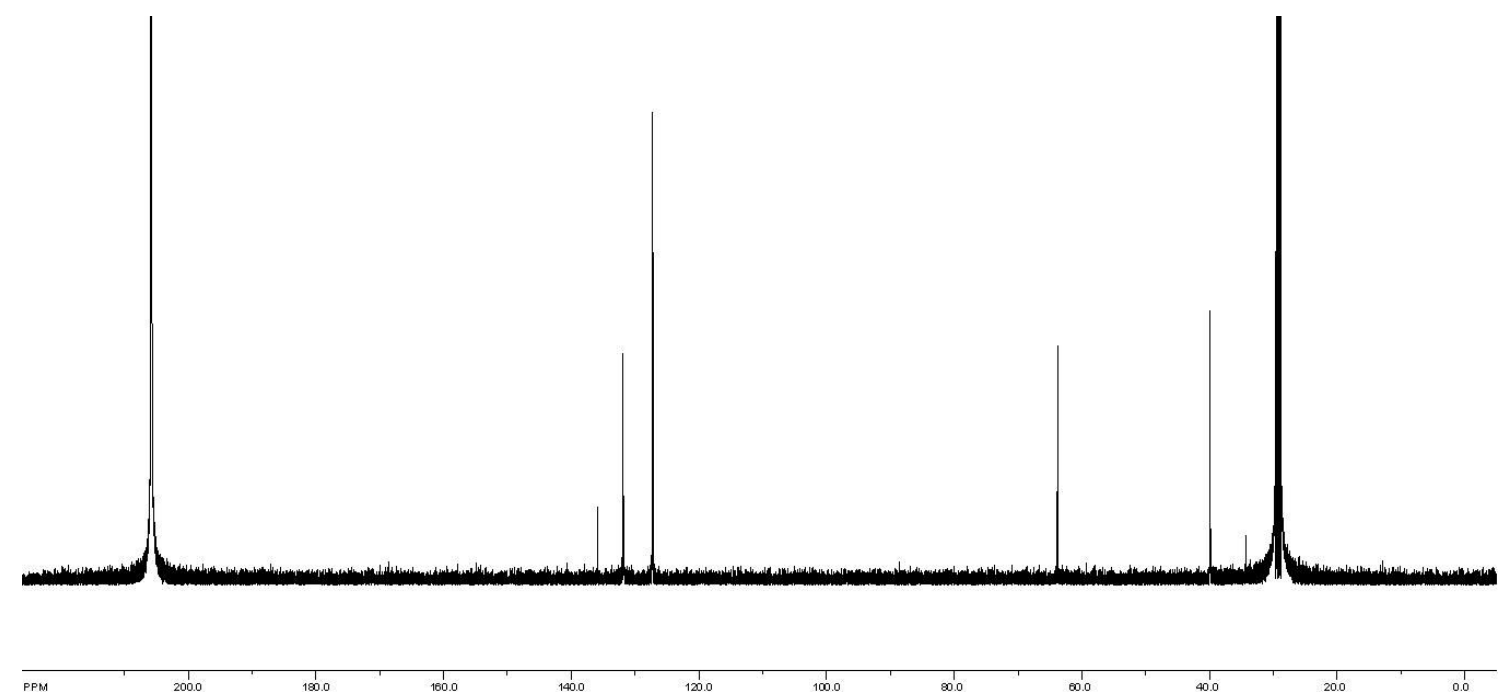

$126 \mathrm{MHz}{ }^{13} \mathrm{C}$ NMR of Compound (14) in Acetone- $d_{6}$

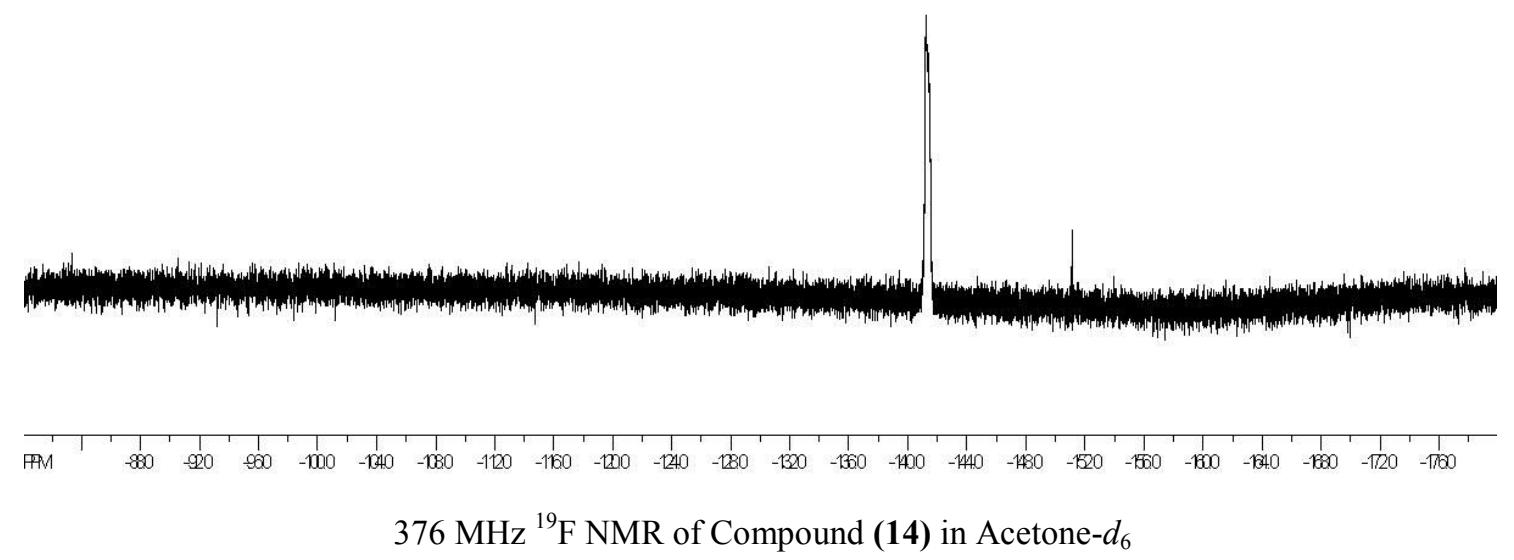




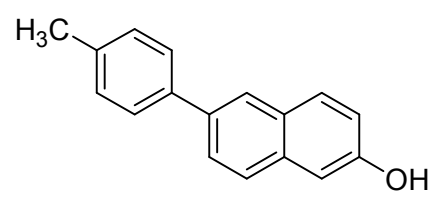

(15)

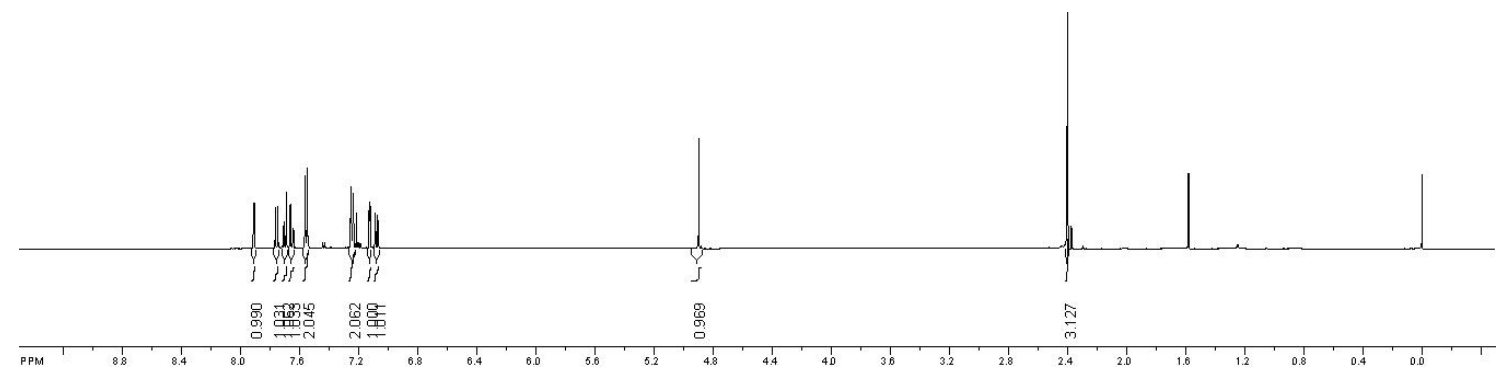

$500 \mathrm{MHz}{ }^{1} \mathrm{H}$ NMR of Compound (15) in $\mathrm{CDCl}_{3}$

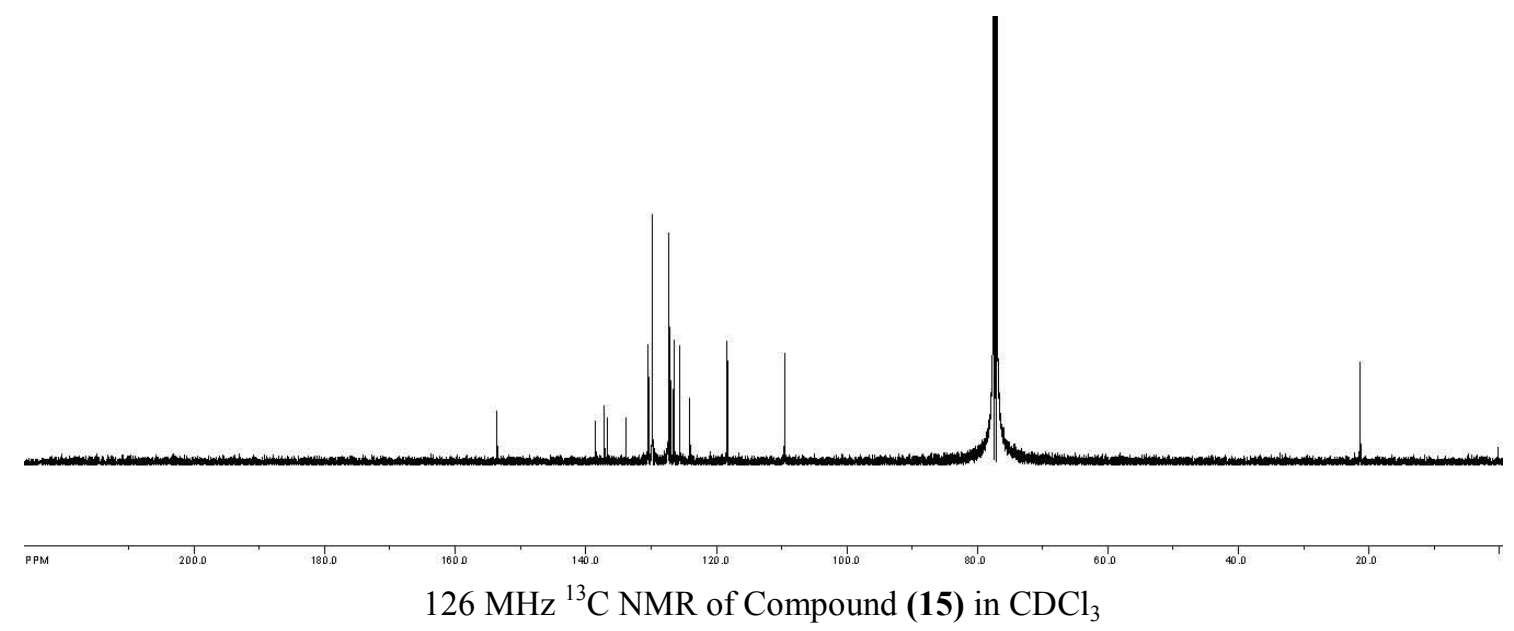




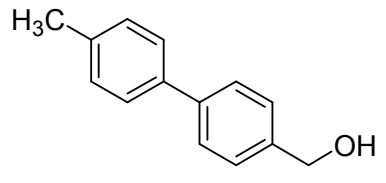

(16)
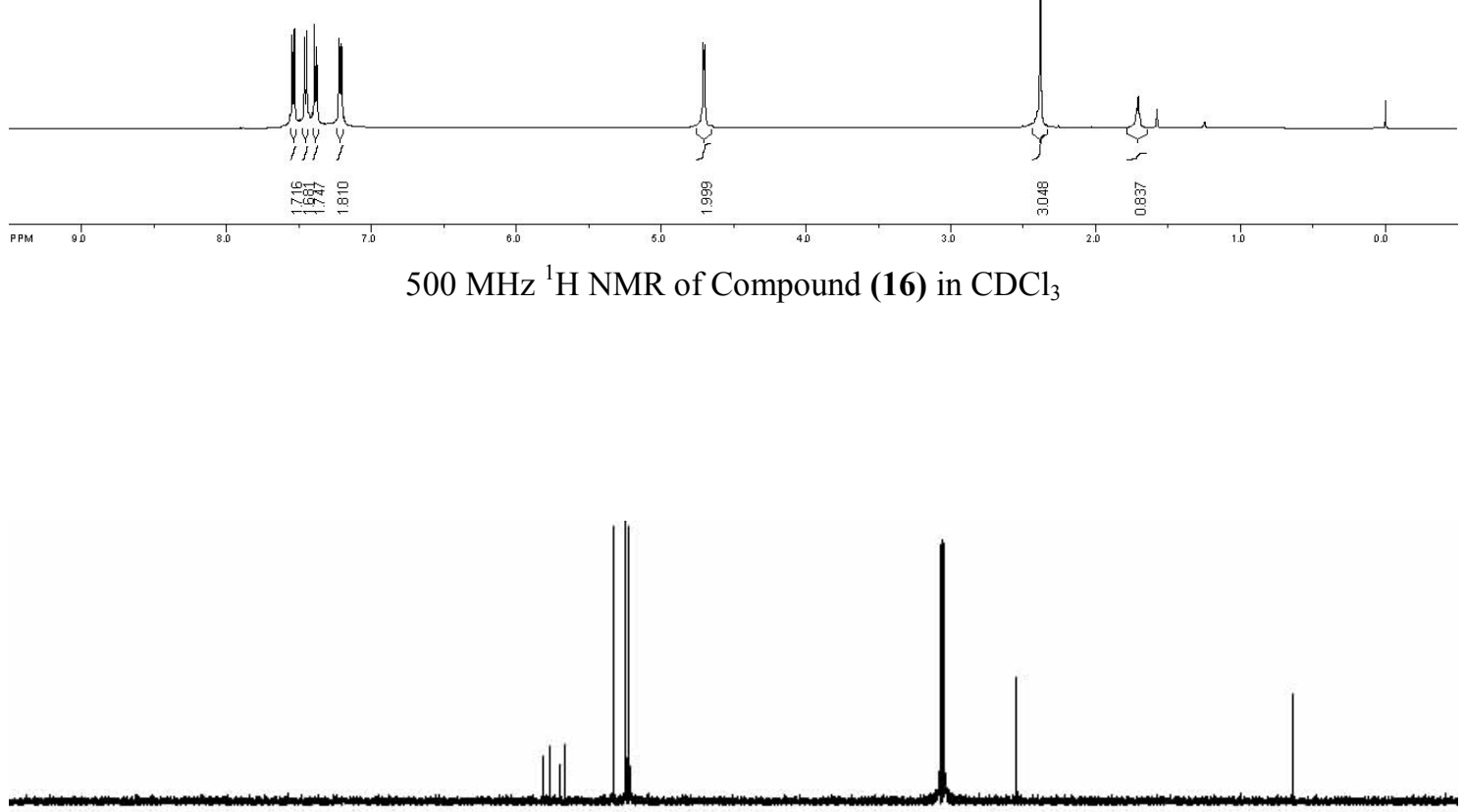

PPM

$\operatorname{con}$

เin

100

काव

mo

כख्य

gat

$126 \mathrm{MHz}{ }^{13} \mathrm{C}$ NMR of Compound (16) in $\mathrm{CDCl}_{3}$

S27 

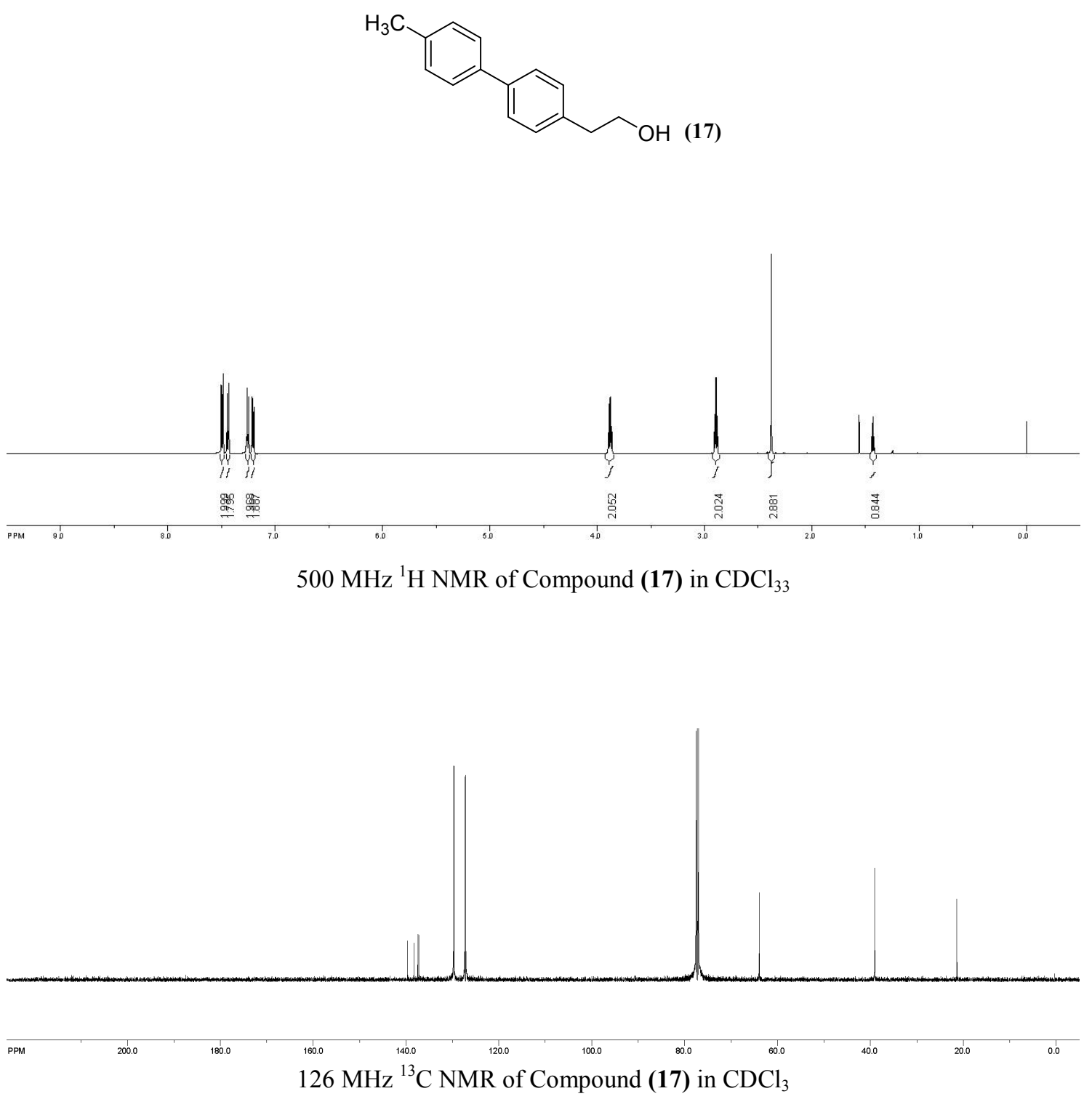

S28 

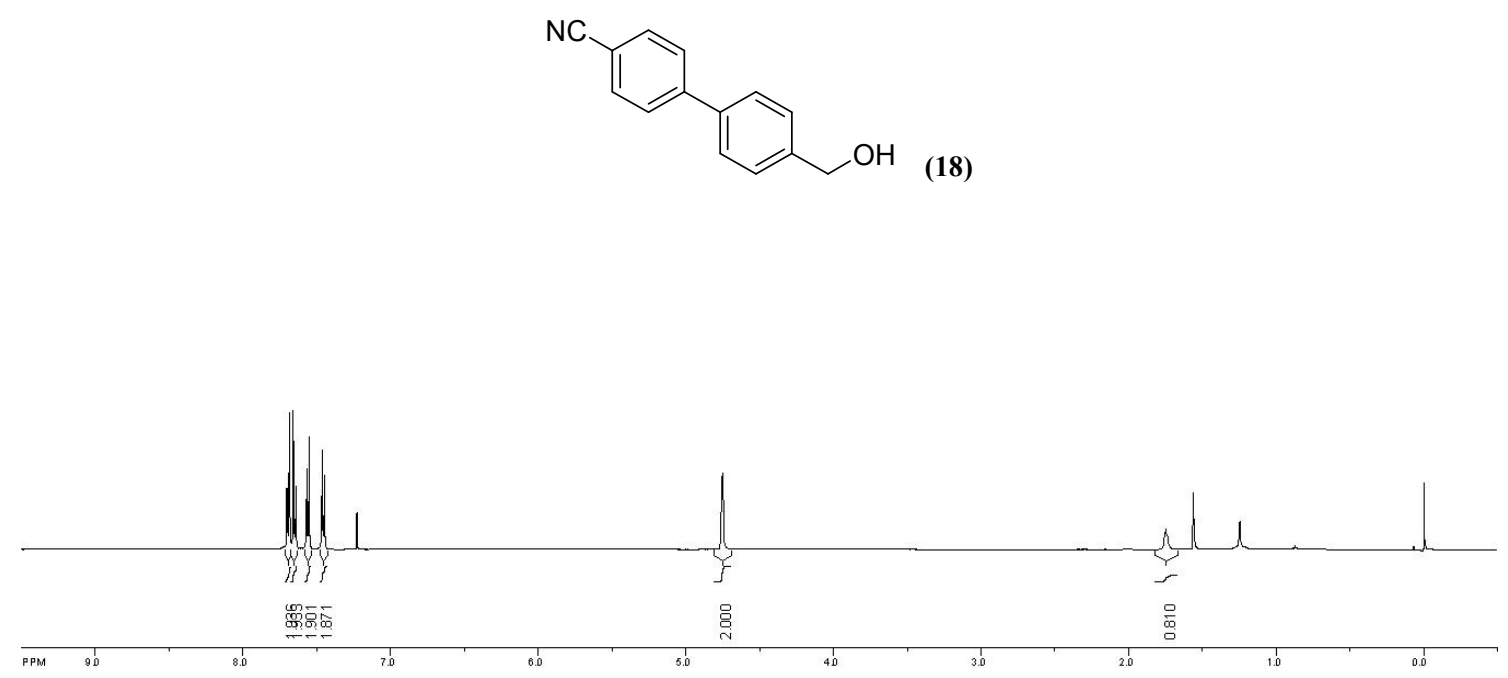

$500 \mathrm{MHz}{ }^{1} \mathrm{H}$ NMR of Compound (18) in $\mathrm{CDCl}_{3}$

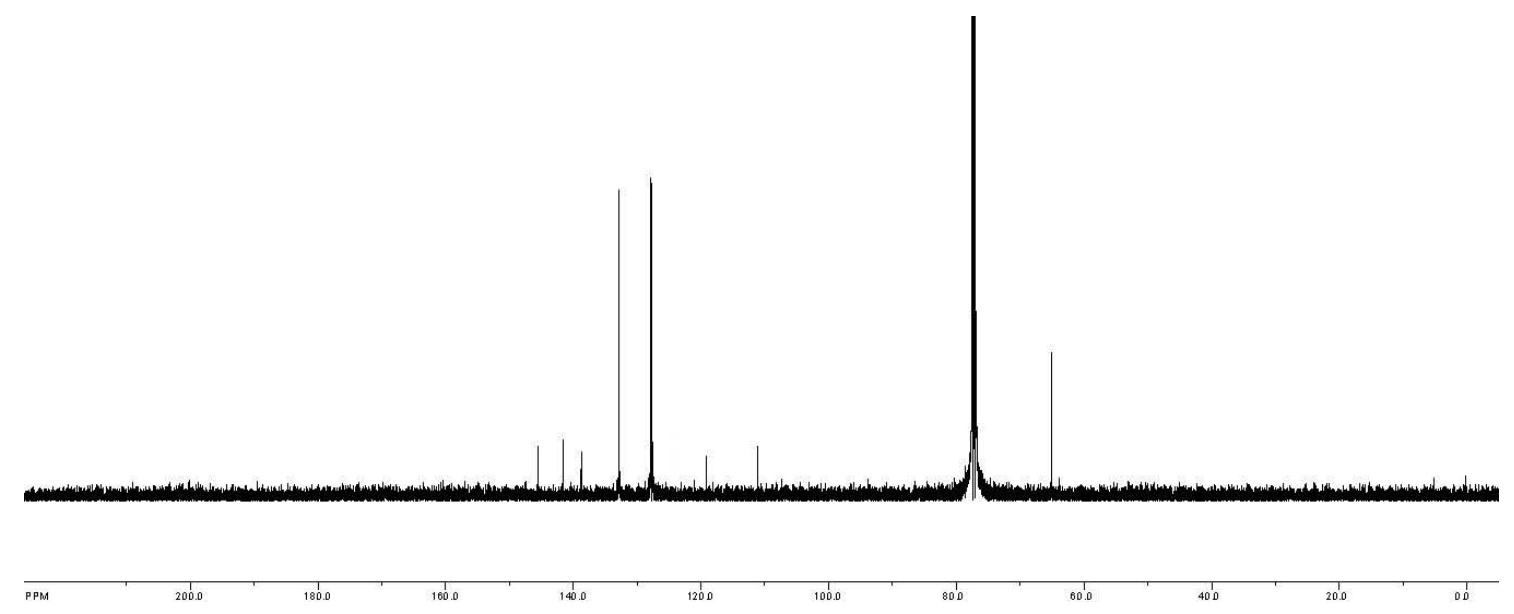

$126 \mathrm{MHz}{ }^{13} \mathrm{C}$ NMR of Compound (18) in $\mathrm{CDCl}_{3}$ 

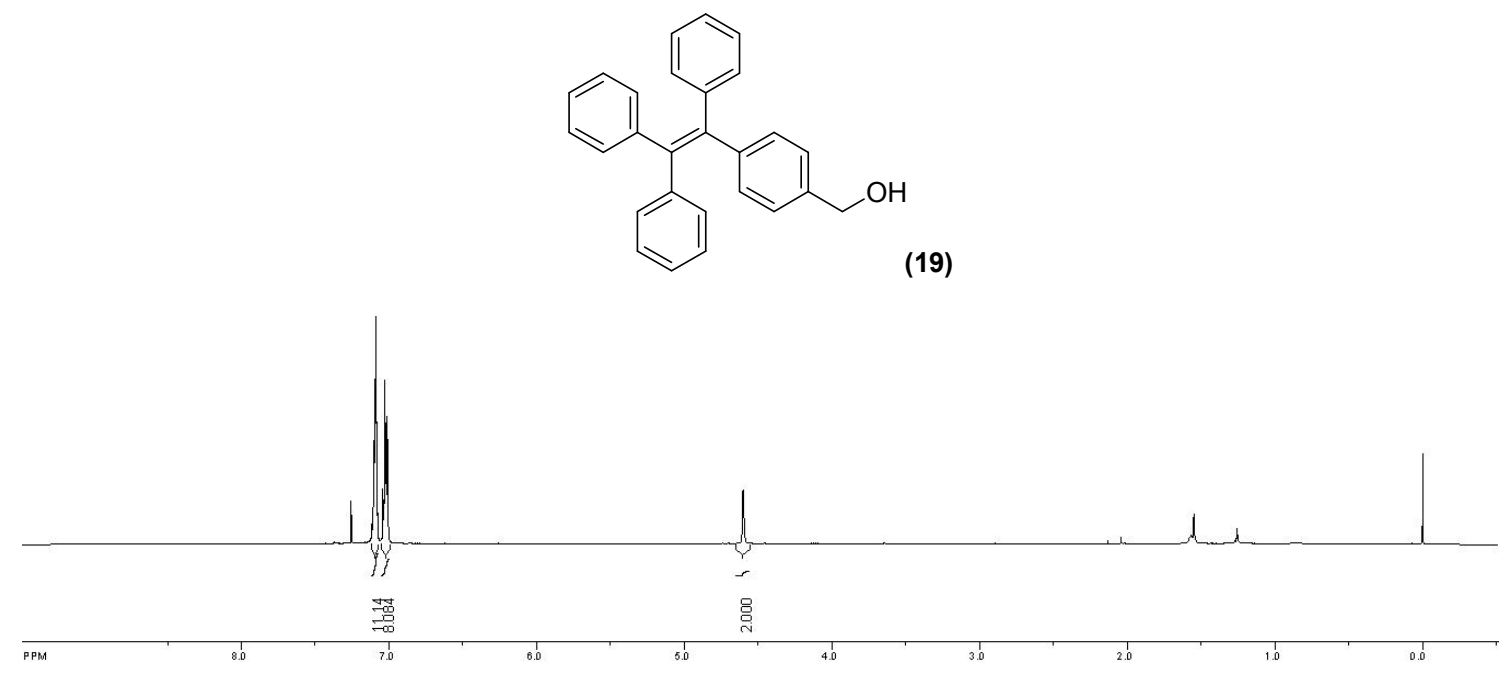

$500 \mathrm{MHz}{ }^{1} \mathrm{H}$ NMR of Compound (19) in $\mathrm{CDCl}_{3}$

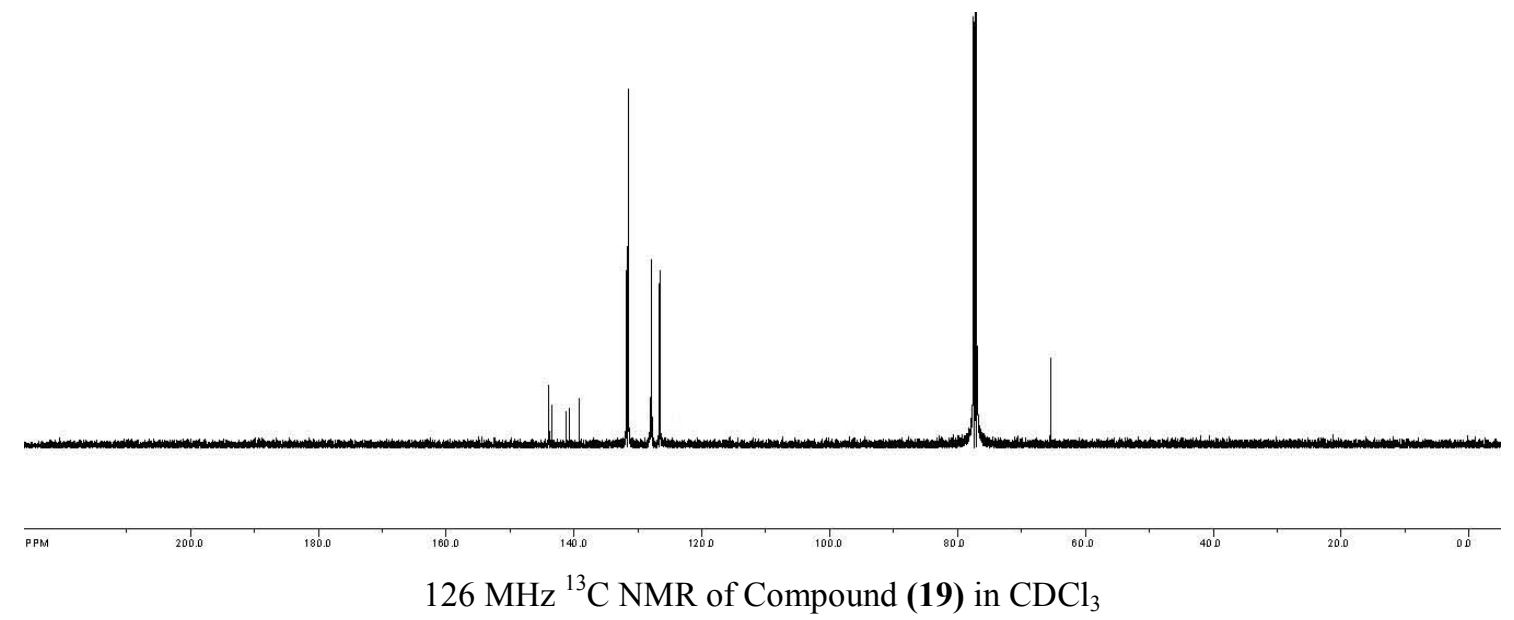

